\title{
UNIFORMLY EXPONENTIALLY STABLE APPROXIMATIONS FOR A CLASS OF SECOND ORDER EVOLUTION EQUATIONS
}

\author{
APPLICATION TO LQR PROBLEMS
}

\author{
Karim RAmdani $^{1,2}$, TAKÉo TAKahashi ${ }^{1,2}$ And Marius TuCsnaK ${ }^{1,2}$
}

\begin{abstract}
We consider the approximation of a class of exponentially stable infinite dimensional linear systems modelling the damped vibrations of one dimensional vibrating systems or of square plates. It is by now well known that the approximating systems obtained by usual finite element or finite difference are not, in general, uniformly stable with respect to the discretization parameter. Our main result shows that, by adding a suitable numerical viscosity term in the numerical scheme, our approximations are uniformly exponentially stable. This result is then applied to obtain strongly convergent approximations of the solutions of the algebraic Riccati equations associated to an LQR optimal control problem. We next give an application to a non-homogeneous string equation. Finally we apply similar techniques for approximating the equations of a damped square plate.
\end{abstract}

Mathematics Subject Classification. 93D15, 65M60, 65M12.

Received September 21, 2005. Revised January 19, 2006.

Published online June 5, 2007.

\section{IntRodUCtion AND STATEMENT OF THE MAIN RESUlts}

Let $H$ and $U$ be real Hilbert spaces, let $A_{0}: \mathcal{D}\left(A_{0}\right) \rightarrow H$ be a self-adjoint, positive operator with $A_{0}^{-1}$ compact in $H$ and let $B_{0} \in \mathcal{L}(U, H)$ be a control operator. The inner product in $H$ is denoted by $\langle\cdot, \cdot\rangle$ and $\|\cdot\|$ stands for the corresponding norm. We consider the system described by

$$
\begin{gathered}
\ddot{w}(t)+A_{0} w(t)=B_{0} u(t), \\
w(0)=w_{0}, \quad \dot{w}(0)=w_{1}
\end{gathered}
$$

where $t \in[0, \infty)$ is the time. Most of the linear equations modelling the vibrations of elastic structures with distributed control can be written in the form (1.1), where $w$ stands for the displacement field.

We define the energy at instant $t$ by

$$
E(t)=\frac{1}{2}\left\{\|\dot{w}(t)\|^{2}+\left\|A_{0}^{\frac{1}{2}} w(t)\right\|^{2}\right\}
$$

\footnotetext{
Keywords and phrases. Uniform exponential stability, LQR optimal control problem, wave equation, plate equation, finite element, finite difference.

1 Institut Elie Cartan University of Nancy-I, POB 239, Vandœuvre-les-Nancy 54506, France; marius.tucsnak@loria.fr

2 INRIA Lorraine, Projet CORIDA.
} 
Simple formal calculations show that

$$
E(0)-E(t)=-\int_{0}^{t}\left\langle B_{0} u(s), \dot{w}(s)\right\rangle \mathrm{d} s, \quad \forall t \geqslant 0 .
$$

The above relation suggests the use of the input $u$ given in the feedback form $u(t)=-B_{0}^{*} \dot{w}(t)$, which obviously gives a non increasing energy and which corresponds to collocated actuators and sensors. We obtain in this way the closed loop system

$$
\begin{gathered}
\ddot{w}(t)+A_{0} w(t)+B_{0} B_{0}^{*} \dot{w}(t)=0 \\
w(0)=w_{0}, \dot{w}(0)=w_{1} .
\end{gathered}
$$

In this paper we assume that the above system is exponentially stable, i.e., there exist $M, \alpha>0$ such that $E(t) \leqslant M \mathrm{e}^{-\alpha t} E(0)$ for all $t \geqslant 0$. Our aim is to construct finite dimensional systems satisfying the following conditions:

(1) They approximate the system (1.3)-(1.4) in a sense which will be made precise later.

(2) They are uniformly exponentially stable, i.e., they are exponentially stable and their decay to zero when $t \rightarrow \infty$ is uniform with respect to the discretization parameter (the notion of uniform exponential stability will be detailed later).

The problem of constructing finite dimensional systems satisfying the above conditions is not obvious because of the existence of spurious high frequency modes. Indeed, by applying the standard finite difference or finite element space semi-discretizations, these spurious modes are only weakly damped. Therefore the corresponding approximation of the controlled wave equation (even in one space dimension) is not uniformly exponentially stabilizable (or uniformly exactly controllable).

Several remedies have been proposed to overcome this difficulty: Tychonoff regularization in Glowinski, Li and Lions [8], mixed finite elements in Banks, Ito and Wang [2], filtering of high frequencies in Infante and Zuazua [10]. We refer to the review paper Zuazua [20] for more details and extensive references.

In this work, in order to damp the spurious modes, we use a "numerical viscosity" in our approximation schemes of Galerkin type. This idea has been introduced, for the approximation of the homogeneous string equation with distributed damping, in Tcheugoué Tébou and Zuazua [18]. The main novelties brought in by this work are the following:

- We give general conditions on the spectral elements of $A_{0}$ and on $B_{0}$ (i.e., on the continuous problem only) ensuring the uniform exponential stability of the approximating systems. In particular, we do not need the analytic expressions of eigenelements of the approximated problem (which are, in general, unknown). Our assumption on the existence of a gap between eigenvalues restricts the range of direct applications of our main result to PDE's in one space dimension. However, unlike results in the existing literature, we are able to tackle the case of variable coefficients. Moreover, similar ideas (based on frequency domain techniques) can be applied to some problems in two space dimensions (see the example in Sect. 7).

- We prove that the schemes with numerical viscosity yield strongly convergent approximations of the Riccati operator solving an LQR optimal control problem associated to the system (1.1)-(1.2).

- Our methods and results can be applied to a wide class of systems governed by partial differential equations including non-homogeneous elastic strings, elastic beams or elastic plates.

In order to give the precise statement of the main result, we introduce several notations and we make some assumptions.

Let $\mathcal{D}\left(A_{0}^{\frac{1}{2}}\right)$ be the completion of $\mathcal{D}\left(A_{0}\right)$ with respect to the norm

$$
\|\varphi\|_{\frac{1}{2}}=\sqrt{\left\langle A_{0} \varphi, \varphi\right\rangle} \quad \forall \varphi \in \mathcal{D}\left(A_{0}\right)
$$


and assume that $\left(V_{h}\right)_{h>0}$ is a sequence of finite dimensional subspaces of $\mathcal{D}\left(A_{0}^{\frac{1}{2}}\right)$. For every $h>0$ we denote by $N(h)$ the dimension of $V_{h}$. The inner product in $V_{h}$ is the restriction of the inner product on $H$ and it is still denoted by $\langle\cdot, \cdot\rangle$. We define the linear operator $A_{0 h} \in \mathcal{L}\left(V_{h}\right)$ by

$$
\left\langle A_{0 h} \varphi_{h}, \psi_{h}\right\rangle=\left\langle A_{0}^{\frac{1}{2}} \varphi_{h}, A_{0}^{\frac{1}{2}} \psi_{h}\right\rangle \quad \forall \varphi_{h}, \psi_{h} \in V_{h} .
$$

The operator $A_{0 h}$ is clearly symmetric and positive-definite. We also consider a sequence of subspaces $\left(U_{h}\right)$ of $U$ and we define the operators $B_{0 h} \in \mathcal{L}\left(U_{h}, V_{h}\right)$ by

$$
B_{0 h} u_{h}=\widetilde{\pi}_{h} B_{0} u_{h} \quad \forall u_{h} \in U_{h}
$$

where $\widetilde{\pi}_{h}$ is the orthogonal projection of $H$ onto $V_{h}$. The adjoint $B_{0 h}^{*}$ of $B_{0 h}$ is then given by the relation

$$
B_{0 h}^{*} \varphi_{h}=\rho_{h} B_{0}^{*} \varphi_{h} \quad \forall \varphi_{h} \in V_{h},
$$

where $\rho_{h}$ is the orthogonal projection of $U$ onto $U_{h}$. The above assumptions imply that the sequences $\left(\left\|B_{0 h}\right\|_{\mathcal{L}\left(U_{h}, V_{h}\right)}\right)_{h \in\left(0, h^{*}\right)}$ and $\left(\left\|B_{0 h}^{*}\right\|_{\mathcal{L}\left(V_{h}, U_{h}\right)}\right)_{h \in\left(0, h^{*}\right)}$ are bounded, for any $h^{*}>0$.

We also suppose that the family of spaces $\left(V_{h}\right)$ (respectively $U_{h}$ ) approximates the space $\mathcal{D}\left(A_{0}^{\frac{1}{2}}\right)$ (respectively $U$ ). More precisely, if $\pi_{h}$ denotes the orthogonal projection of $\mathcal{D}\left(A_{0}^{\frac{1}{2}}\right)$ onto $V_{h}$, we suppose that there exist $\theta>0, h^{*}>0$ and $C_{0}>0$ such that, for all $h \in\left(0, h^{*}\right)$, we have:

together with

$$
\begin{aligned}
\left\|\pi_{h} \varphi-\varphi\right\|_{\frac{1}{2}} \leqslant C_{0} h^{\theta}\left\|A_{0} \varphi\right\| & \forall \varphi \in \mathcal{D}\left(A_{0}\right), \\
\left\|\pi_{h} \varphi-\varphi\right\| \leqslant C_{0} h^{2 \theta}\left\|A_{0} \varphi\right\| & \forall \varphi \in \mathcal{D}\left(A_{0}\right),
\end{aligned}
$$

Note that the density of $\mathcal{D}\left(A_{0}\right)$ in $H$ and (1.7) imply that

$$
\lim _{h \rightarrow 0} \tilde{\pi}_{h} \varphi=\varphi \quad \text { in } H \quad \forall \varphi \in H .
$$

Assumptions (1.7) and (1.8) are, in particular, satisfied when using finite elements for the approximation of Sobolev spaces.

The main result of this paper is

Theorem 1.1. Suppose that

(1) $A_{0}^{\frac{1}{2}}$ has simple eigenvalues

and there exists a constant $\gamma_{0}>0$ such that

$$
\lambda_{1}<\ldots<\lambda_{n}<\ldots
$$

$$
\lambda_{n+1}-\lambda_{n} \geqslant \gamma_{0} \quad \forall n \in \mathbb{N} .
$$

(2) There exists a constant $\beta_{0}>0$ such that

$$
\left\|B_{0}^{*} \varphi\right\|_{U} \geqslant \beta_{0}
$$

for all normalized (in $H$ ) eigenvector $\varphi$ of $A_{0}^{\frac{1}{2}}$. 
(3) The families of subspaces $\left(V_{h}\right)$ and $\left(U_{h}\right)$ satisfy $(1.7)-(1.10)$.

Then the family of systems

$$
\begin{gathered}
\ddot{w}_{h}(t)+A_{0 h} w_{h}(t)+B_{0 h} B_{0 h}^{*} \dot{w}_{h}(t)+h^{\theta} A_{0 h} \dot{w}_{h}(t)=0, \\
w_{h}(0)=w_{0 h} \in V_{h}, \quad \dot{w}_{h}(0)=w_{1 h} \in V_{h},
\end{gathered}
$$

is uniformly exponentially stable, in the sense that there exist constants $M, \alpha, h^{*}>0$ (independent of $h, w_{0 h}$ and $\left.w_{1 h}\right)$ such that for all $h \in\left(0, h^{*}\right)$ :

$$
\left\|\dot{w}_{h}(t)\right\|^{2}+\left\|A_{0 h}^{\frac{1}{2}} w_{h}(t)\right\|^{2} \leqslant M \mathrm{e}^{-\alpha t}\left(\left\|w_{1 h}\right\|^{2}+\left\|A_{0 h}^{\frac{1}{2}} w_{0 h}\right\|^{2}\right) \quad \forall t \geqslant 0 .
$$

Remark 1.2. The assumption that $B_{0}$ is bounded forbids the applications to PDE systems controlled from the boundary. The removal of this restriction, which would be very important for applications, looks difficult for at least two reasons:

- The frequency domain techniques we use are not available in the case of unbounded input operators.

- The approximation theory for LQR problems (applied in Sect. 5) is also unavailable in the case of the wave equation with boundary control.

Remark 1.3. According to [3], assumptions (1.12) and (1.13) imply that (1.1) (1.2) is exponentially stable.

The outline of this paper is the following. In Section 2, we derive some properties satisfied by the eigenelements of the discretized problem. In Section 3 we state and prove three technical lemmas. These lemmas are used in Section 4 for the proof of our main result (Th. 1.1). The uniform exponential stability result in Theorem 1.1 is then used in Section 5 to approximate the Riccati operators associated to a class of LQR problems. In Section 6, we apply our general results to a system modelling the vibrations of a non homogeneous string. The last section is devoted to another application of the three lemmas in Section 3: the finite difference approximation of the plate equation in a square.

\section{Spectral AnAlysis of the Discretized PROBlem}

In this section, we first give a result on the Galerkin approximation of the "low frequency" eigenvalues of a self-adjoint operator.

Lemma 2.1. Let $A_{0}: \mathcal{D}\left(A_{0}\right) \rightarrow H$ be a self-adjoint, positive operator with $A_{0}^{-1}$ compact in $H$ and let

$$
0<\lambda_{1} \leqslant \cdots \leqslant \lambda_{n} \leqslant \ldots
$$

be the eigenvalues of $A_{0 h}^{\frac{1}{2}}$. Suppose that the family of spaces $\left(V_{h}\right)$ satisfies assumption (1.8) and let

$$
0<\lambda_{1, h} \leqslant \cdots \leqslant \lambda_{N(h)}
$$

be the eigenvalues of $A_{0 h}^{\frac{1}{2}}$, with $A_{0 h}$ defined by (1.5). Then

$$
\lambda_{n} \leqslant \lambda_{n, h} \quad \forall n \in\{1,2, \ldots, N(h)\} .
$$

Moreover, there exist positive constants $\varepsilon, h^{*}$ and $C$, such that, for all $0<h<h^{*}$ and for all $n \in\{1,2, \ldots, N(h)\}$, satisfying

we have

$$
h^{\theta} \lambda_{n}^{2} \leqslant \varepsilon,
$$

$$
\lambda_{n, h} \leqslant \lambda_{n}+C \varepsilon .
$$


Proof. First, we recall that the Min-Max principle (see, for instance, [16], Chap. 6) implies (2.1). Denote

$$
\sigma_{n, h}=\max _{\varphi \in \mathcal{B}_{n}}\left|2\left\langle\varphi, \varphi-\pi_{h} \varphi\right\rangle-\left\|\varphi-\pi_{h} \varphi\right\|^{2}\right|
$$

where $\mathcal{B}_{n}=\left\{\varphi \in E_{n},\|\varphi\|=1\right\}$ and where $E_{n}$ is the subspace spanned by the $n$ first eigenvectors of $A_{0}^{\frac{1}{2}}$. According to a result in [17], p. 229, we have that

$$
\lambda_{n, h} \leqslant \frac{\lambda_{n}}{\sqrt{1-\sigma_{n, h}}} \quad \forall n \in\{1,2, \ldots, N(h)\} .
$$

provided that $\sigma_{n, h}<1$. Let us show that $\varepsilon$ in $(2.2)$ can be chosen such that $\sigma_{n, h}<\frac{1}{2}$, for all $n \in\{1, \ldots, N(h)\}$ satisfying (2.2). Since $\varphi \in \mathcal{B}_{n}$, by using (1.8) we obtain that the first term in the definition (2.4) of $\sigma_{n, h}$ satisfies:

$$
2\left|\left\langle\varphi, \varphi-\pi_{h} \varphi\right\rangle\right| \leqslant 2\left\|\varphi-\pi_{h} \varphi\right\| \leqslant 2 C_{0} h^{2 \theta} \lambda_{n}^{2}
$$

By using again (1.8) and the fact that $\varphi \in B_{n}$ we get that

$$
\left\|\varphi-\pi_{h} \varphi\right\|^{2} \leqslant C_{0}^{2} h^{4 \theta}\left\|A_{0} \varphi\right\|^{2} \leqslant C_{0}^{2}\left(h^{2 \theta} \lambda_{n}^{2}\right)^{2}
$$

Consequently, for $\varepsilon<2 / C_{0}$ and $h^{*}<1$, the second term in the definition (2.4) of $\sigma_{n, h}$ is smaller than the first one, so we have

$$
0 \leqslant \sigma_{n, h} \leqslant 4\left|\left\langle\varphi, \varphi-\pi_{h} \varphi\right\rangle\right| \leqslant 4 C_{0} h^{\theta}\left(h^{\theta} \lambda_{n}^{2}\right) \leqslant 4 C_{0} \varepsilon
$$

Thus, if we assume that $\varepsilon \leqslant 1 /\left(8 C_{0}\right)$, then we have indeed that $\sigma_{n, h} \leqslant \frac{1}{2}$ for all $n \in\{1, \ldots, N(h)\}$ satisfying $(2.2)$.

Consequently

$$
\lambda_{n, h} \leqslant \frac{\lambda_{n}}{\sqrt{1-\sigma_{n, h}}} \leqslant \lambda_{n}\left(1+\sqrt{2 \sigma_{n, h}}\right) \leqslant \lambda_{n}\left(1+\sqrt{8 C_{0}} h^{\theta} \lambda_{n}\right) \leqslant \lambda_{n}+C \varepsilon
$$

for all $n \in\{1,2, \ldots, N(h)\}$ and for all $h<1$ satisfying $(2.2)$, with $C=\sqrt{8 C_{0}}$.

We next show that the gap condition (1.12) and the observability condition (1.13) which are the basic assumptions in Theorem 1.1 still hold for the approximate problem (uniformly in $h$ ), provided that we consider only "low frequencies". More precisely, the following result holds:

Proposition 2.2. With the notation in Lemma 2.1 and under the assumptions of Theorem 1.1, there exist two constants $\varepsilon>0$ and $h^{*}>0$, such that, for all $0<h<h^{*}$ and for all $n \in\{1,2, \ldots, N(h)\}$, satisfying

$$
h^{\theta} \lambda_{n}^{2} \leqslant \varepsilon
$$

we have

(1) the eigenvalues $\lambda_{n, h}$ are simple, and there exists a constant $\gamma>0$ (independent of $h$ ) such that

$$
\lambda_{n+1, h}-\lambda_{n, h} \geqslant \gamma
$$

(2) there exists a constant $\beta>0$ (independent of $h$ ) such that for every integer $n$ satisfying (2.7) and for every normalized (in $H$ ) eigenvector $\varphi_{n, h}$ of $A_{0 h}^{\frac{1}{2}}$ associated with the eigenvalue $\lambda_{n, h}$, there holds

$$
\left\|B_{0 h}^{*} \varphi_{h}\right\|_{U} \geqslant \beta
$$


Proof. By using Lemma 2.1 we see that for all $n \in\{1,2, \ldots, N(h)\}$ satisfying (2.7) we have

$$
\lambda_{n+1, h}-\lambda_{n, h} \geqslant \lambda_{n+1}-\lambda_{n}-C \varepsilon \geqslant \gamma_{0}-C \varepsilon .
$$

The above relation implies that the uniform gap condition (2.8) with $\gamma=\frac{\gamma_{0}}{2}$ holds provided that

$$
\varepsilon \leqslant \gamma_{0} /(2 C)
$$

From now on, we will assume that (2.10) is satisfied.

Consider $\left(\phi_{n}\right)_{n \geqslant 1}$ an orthonormal basis of $H$ constituted by eigenvectors of $A_{0}^{\frac{1}{2}}$ associated with the eigenvalues $\left(\lambda_{n}\right)_{n \geqslant 1}$. Similarly, let $\left(\varphi_{n, h}\right)_{1 \leqslant n \leqslant N(h)}$ be an orthonormal basis of $V_{h}$ constituted by eigenvectors of $A_{0 h}^{\frac{1}{2}}$ associated with the eigenvalues $\left(\lambda_{n, h}\right)_{1 \leqslant n \leqslant N(h)}$.

In order to show that (2.9) holds, we need to obtain an error estimate for the eigenvectors $\varphi_{n, h}$. We first recall that, according to a classical result for the Galerkin approximation of eigenvectors (see for instance [16], p. 149), we have:

where we have set: $\rho_{n, h}=\max _{\substack{m \leqslant N(h) \\ m \neq n}} \frac{\phi_{n}-\varphi_{n, h}\left\|\leqslant 2\left(1+\rho_{n, h}\right)\right\| \phi_{n}-\pi_{h} \phi_{n} \|,}{\left|\lambda_{m, h}-\lambda_{n}\right|}$. By (1.7), we get that

$$
\left\|\phi_{n}-\varphi_{n, h}\right\| \leqslant 2 C_{0}\left(1+\rho_{n, h}\right) h^{2 \theta} \lambda_{n}^{2} .
$$

It remains to obtain a bound for $\rho_{n, h}$, for $n \in\{1,2, \ldots, N(h)\}$ satisfying (2.7). For the rest of the proof, let us fix such an integer $n$.

First, we notice that if $1 \leqslant m \leqslant N(h)$ does not satisfy (2.7), then $m>n$, and thus, using (2.1) and the gap condition (1.12), we get that

$$
\lambda_{m, h}-\lambda_{n} \geqslant \lambda_{m}-\lambda_{n} \geqslant \gamma_{0} .
$$

On the other hand, if $1 \leqslant m \leqslant N(h)$, with $m \neq n$, satisfies (2.7), then by (2.6) and (2.10), we have

$$
\left|\lambda_{m, h}-\lambda_{n}\right| \geqslant\left|\lambda_{m}-\lambda_{n}\right|-\left|\lambda_{m, h}-\lambda_{m}\right| \geqslant \gamma_{0}-c \varepsilon \geqslant \gamma_{0} / 2,
$$

Consequently, for all $m \in\{1,2, \ldots, N(h)\}$, with $m \neq n$ we have

$$
\left|\lambda_{m, h}-\lambda_{n}\right| \geqslant \gamma_{0} / 2
$$

By the above inequality and the definition of $\rho_{n, h}$, we get

$$
\rho_{n, h} \leqslant 2 \frac{\lambda_{n}}{\gamma_{0}}
$$

Thus, plugging this relation into (2.11), we obtain that the inequalities

$$
\left\|\phi_{n}-\varphi_{n, h}\right\| \leqslant 2 C_{0} h^{2 \theta} \lambda_{n}^{2}+\frac{4 C_{0}}{\gamma_{0}} h^{2 \theta} \lambda_{n}^{3} \leqslant 2 C_{0} \varepsilon+\frac{4 C_{0}}{\gamma_{0}} \varepsilon^{3 / 2},
$$

hold for all $0<h<1$. This relation shows that there exists $C_{1}>0$ such that

$$
\left\|\phi_{n}-\varphi_{n, h}\right\| \leqslant C_{1} \varepsilon
$$


On the other hand,

$$
B_{0 h}^{*} \varphi_{n, h}=\rho_{h} B_{0}^{*} \varphi_{n, h}=B_{0}^{*} \phi_{n}+\left(\rho_{h} B_{0}^{*}-B_{0}^{*}\right) \phi_{n}+\rho_{h} B_{0}^{*}\left(\varphi_{n, h}-\phi_{n}\right)
$$

and by (1.10), we get that

$$
\left\|\rho_{h} B_{0}^{*} \phi_{n}-B_{0}^{*} \phi_{n}\right\| \leqslant C_{0} h^{2 \theta} \lambda_{n}^{2} \leqslant C_{0} \varepsilon
$$

Equations (1.13), (2.13) and (2.15) imply that (2.9) holds for some $\beta>0$ independent of $h$.

\section{THREE LEMMAS}

In this section we give three results which will be essentially used in the proof of Theorem 1.1. However, since we will also apply these results for the the finite difference approximation in Section 7, we use assumptions which are weaker than those in Theorem 1.1. More precisely, throughout this section we make the following assumptions

[H1] $\left(V_{h}\right)$ (respectively $\left(U_{h}\right)$ ) is a family of finite dimensional normed spaces with norm denoted by $\|\cdot\|_{V_{h}}$ (respectively by $\|\cdot\|_{U_{h}}$ ).

[H2] $\left(A_{0 h}\right) \subset \mathcal{L}\left(V_{h}\right)$ is a family of self-adjoint and positive-definite operators such that there exists a constant $m_{0}>0$ with $\left\|A_{0 h}\right\|_{\mathcal{L}\left(V_{h}\right)} \geqslant m_{0}>0$, for all $h \in\left(0, h^{*}\right)$.

[H3] $\left(B_{0 h}\right) \subset \mathcal{L}\left(U_{h}, V_{h}\right)$ is a family of linear operators such that there exists a constant $m_{1}>0$ with $\left\|B_{0 h}\right\|_{\mathcal{L}\left(U_{h}, V_{h}\right)} \leqslant m_{1}$, for all $h \in\left(0, h^{*}\right)$.

We introduce the Hilbert space $X_{h}=V_{h} \times V_{h}$ endowed with the norm

$$
\left\|\left[\begin{array}{c}
\varphi_{h} \\
\psi_{h}
\end{array}\right]\right\|_{X_{h}}^{2}=\left\|A_{0 h}^{\frac{1}{2}} \varphi_{h}\right\|_{V_{h}}^{2}+\left\|\psi_{h}\right\|_{V_{h}}^{2} .
$$

The system (1.14)-(1.15) is equivalent to the following first order system in $X_{h}$ :

$$
\dot{z}_{h}(t)=\widetilde{A}_{h} z_{h}(t), \quad z_{h}(0)=z_{0 h},
$$

where

$$
\widetilde{A}_{h}=\left[\begin{array}{ll}
0 & I \\
-A_{0 h} & -h^{\theta} A_{0 h}-B_{0 h} B_{0 h}^{*}
\end{array}\right], \quad z_{0 h}=\left[\begin{array}{l}
w_{0 h} \\
w_{1 h}
\end{array}\right] .
$$

Lemma 3.1. Suppose that $\left(V_{h}\right),\left(U_{h}\right), A_{0 h}$ and $B_{0 h}$ satisfy assumption [H1]-[H3]. Then the spectrum of the operator $\widetilde{A}_{h}$, defined in (3.17), contains no point on the imaginary axis.

Proof. Suppose that $\left[\begin{array}{l}\varphi_{h} \\ \psi_{h}\end{array}\right] \in X_{h}=V_{h} \times V_{h}$ and $\omega \in \mathbb{R}$ are such that

$$
\widetilde{A}_{h}\left[\begin{array}{l}
\varphi_{h} \\
\psi_{h}
\end{array}\right]=i \omega\left[\begin{array}{l}
\varphi_{h} \\
\psi_{h}
\end{array}\right]
$$

Then, by using the definition (3.17) of $\widetilde{A}_{h}$, we easily obtain that

$$
\left\{\begin{aligned}
\psi_{h} & =i \omega \varphi_{h}, \\
{\left[\omega^{2}-A_{0 h}-i \omega\left(h^{\theta} A_{0 h}+B_{0 h} B_{0 h}^{*}\right)\right] \varphi_{h} } & =0 .
\end{aligned}\right.
$$

By taking the imaginary part of the inner product of the second relation in (3.18) with $\varphi_{h}$ and by using the fact that $A_{0 h}$ is invertible, we get that $\varphi_{h}=0$. Then, by using the first relation in (3.18) we get that $\psi_{h}=0$. Thus, $i \omega$ cannot be an eigenvalue of $\widetilde{A}_{h}$ and hence $i \omega \in \rho\left(\widetilde{A}_{h}\right)$ for all $\omega \in \mathbb{R}$. 
Notice that the above proof uses in an essential way the numerical viscosity term. Without this term, the result still holds but only with additional assumptions on $B_{0 h}$.

Lemma 3.2. Suppose that $\left(V_{h}\right),\left(U_{h}\right), A_{0 h}$ and $B_{0 h}$ satisfy assumptions $[\mathrm{H} 1]-[\mathrm{H} 3]$. Assume that, for all $n \in \mathbb{N}$, there exist $h_{n} \in\left(0, h^{*}\right), \omega_{n} \in \mathbb{R}$, and $z_{n}=\left[\begin{array}{l}\varphi_{n} \\ \psi_{n}\end{array}\right] \in X_{h_{n}}$ such that

$$
\left\|A_{0 h_{n}}^{\frac{1}{2}} \varphi_{n}\right\|_{V_{h_{n}}}^{2}+\left\|\psi_{n}\right\|_{V_{h_{n}}}^{2}=1 \quad \forall n \in \mathbb{N}
$$

and

Then we have

$$
\left\|i \omega_{n} z_{n}-\widetilde{A}_{h_{n}} z_{n}\right\|_{X_{h_{n}}} \rightarrow 0
$$

$$
\begin{gathered}
h_{n}^{\theta}\left\|A_{0 h_{n}}^{\frac{1}{2}} \psi_{n}\right\|_{V_{h_{n}}}^{2}+\left\|B_{0 h_{n}}^{*} \psi_{n}\right\|_{U_{h_{n}}}^{2} \rightarrow 0 . \\
\lim _{n \rightarrow \infty}\left\|A_{0 h_{n}}^{\frac{1}{2}} \varphi_{n}\right\|_{V_{h_{n}}}^{2}=\lim _{n \rightarrow \infty}\left\|\psi_{n}\right\|_{V_{h_{n}}}^{2}=\frac{1}{2} .
\end{gathered}
$$

Proof. Relation (3.21) follows directly from (3.20) by taking the inner product in $X_{h_{n}}$ of $i \omega_{n} z_{n}-\widetilde{A}_{h_{n}} z_{n}$ by $z_{n}$ and by considering only the real part. In order to prove (3.22) we introduce the operator

$$
A_{1 h}=\left[\begin{array}{cc}
0 & I \\
-A_{0 h} & 0
\end{array}\right] \in \mathcal{L}\left(X_{h}\right)
$$

We obviously have

$$
\widetilde{A}_{h}\left[\begin{array}{l}
\varphi_{h} \\
\psi_{h}
\end{array}\right]=A_{1 h}\left[\begin{array}{l}
\varphi_{h} \\
\psi_{h}
\end{array}\right]-\left[\begin{array}{c}
0 \\
h^{\theta} A_{0 h} \psi_{h}+B_{0 h} B_{0 h}^{*} \psi_{h}
\end{array}\right] \quad \forall\left[\begin{array}{c}
\varphi_{h} \\
\psi_{h}
\end{array}\right] \in X_{h} .
$$

By using (3.20), (3.21), (3.24) and the fact that the operators $B_{0 h_{n}}$ are uniformly bounded we obtain that

$$
\left\|i \omega_{n} z_{n}-A_{1 h_{n}} z_{n}+\left[\begin{array}{c}
0 \\
h_{n}^{\theta} A_{0 h_{n}} \psi_{n}
\end{array}\right]\right\|_{X_{h_{n}}} \rightarrow 0
$$

We show now by a contradiction argument that the sequence $\left(\omega_{n}\right)$ contains no subsequence converging to zero. Suppose that such a subsequence exists. For the sake of simplicity, we still denote it by $\left(\omega_{n}\right)$. Then, by looking to the first component of (3.25) and by using (3.19), we obtain that

$$
\left\|A_{0 h_{n}}^{\frac{1}{2}} \psi_{n}\right\|_{V_{h_{n}}} \rightarrow 0
$$

Moreover, by taking the inner product in $V_{h_{n}}$ of the second component of $i \omega_{n} z_{n}-A_{1 h_{n}} z_{n}+\left[\begin{array}{c}0 \\ h_{n}^{\theta} A_{0 h_{n}} \psi_{n}\end{array}\right]$ by $\varphi_{n}$, by using $(3.19),(3.25),(3.26)$, assumption [H2] and the fact that $\left(h_{n}\right)$ is bounded we get that

$$
\left\|A_{0 h_{n}}^{\frac{1}{2}} \varphi_{n}\right\|_{V_{h_{n}}} \rightarrow 0
$$

The above relation, assumption [H2] and (3.26) contradict (3.19), so we have proved that there exists $n_{0} \in \mathbb{N}$ such that the sequence $\left(\left|\omega_{n}\right|\right)_{n \geqslant n_{0}}$ is bounded away from zero. 
We can now prove (3.22). Taking the inner product in $X_{h_{n}}$ of $(3.25)$ by $\frac{1}{\omega_{n}}\left[\begin{array}{c}\varphi_{n} \\ -\psi_{n}\end{array}\right]$, with $n \geqslant n_{0}$, and by considering only the imaginary part, we obtain:

$$
\lim _{n \rightarrow \infty}\left(\left\|A_{0 h_{n}}^{\frac{1}{2}} \varphi_{n}\right\|_{V_{h_{n}}}^{2}-\left\|\psi_{n}\right\|_{V_{h_{n}}}^{2}\right)=0 .
$$

The above relation and (3.19) yield (3.22).

In order to write in a simple way the eigenvalues and the eigenvectors of the operator $A_{1 h}$, defined in (3.23), we extend the definition of $\lambda_{m, h}$ and of $\varphi_{m, h}$ for $m \in\{-1, \ldots,-N(h)\}$ by setting $\lambda_{m, h}=-\lambda_{-m, h}$ and $\varphi_{m, h}=$ $\varphi_{-m, h}$. Then, it can be easily checked that an orthonormal basis of $X_{h}$ formed by eigenvectors of $A_{1 h}$ is given by

$$
\Phi_{m, h}=\frac{1}{\sqrt{2}}\left[\begin{array}{c}
-\frac{i}{\lambda_{m, h}} \varphi_{m, h} \\
\varphi_{m, h}
\end{array}\right], \quad 0<|m| \leqslant N(h)
$$

where $\Phi_{m, h}$ is an eigenvector associated to the eigenvalue $i \lambda_{m, h}$.

Let $\varepsilon$ be a positive constant. For every $h>0$, we define

$$
M(h)=\max \left\{m \in\{1, \ldots, N(h)\} \mid h^{\theta}\left(\lambda_{m}\right)^{2} \leqslant \varepsilon\right\}
$$

if $h^{\theta}\left(\lambda_{1}\right)^{2} \leqslant \varepsilon$ and $M(h)=0$ otherwise. The proof of our main results is essentially based on the decomposition of the spectrum of $A_{0}^{\frac{1}{2}}$ in two parts:

- The eigenvalues $\lambda_{m}$ with $1 \leqslant m \leqslant M\left(h_{n}\right)$, corresponding to "low frequencies", which will be damped by the feedback control law.

- The eigenvalues $\lambda_{m}$ with $m>M\left(h_{n}\right)$, corresponding to "high frequencies", which will be damped by the numerical viscosity term.

With the above notations, the following result holds:

Lemma 3.3. Suppose that the families of operators $\left(\widetilde{A}_{h}\right)$ and $\left(A_{0 h}\right)$ and the sequences $\left(h_{n}\right),\left(\omega_{n}\right),\left(z_{n}\right)$ satisfy the assumptions in Lemma 3.2. Moreover, assume that there exist positive constants $\eta, C$ and $\varepsilon$ such that

$$
\begin{gathered}
\lambda_{m, h} \geqslant \eta \lambda_{m} \quad \forall m \in\left\{M\left(h_{n}\right)+1, \ldots, N\left(h_{n}\right)\right\}, \\
\lambda_{m, h} \leqslant \lambda_{m}+C \varepsilon \quad \forall m \in\left\{1, \ldots, M\left(h_{n}\right)\right\},
\end{gathered}
$$

where $M(h)$ is defined by (3.28). Let $\left(c_{m}^{n}\right)_{0<|m| \leqslant N(h)}$ be the coordinates of $z_{n}$ in the basis $\left(\Phi_{m, h}\right)_{0<|m| \leqslant N(h)}$, i.e.,

$$
z_{n}=\sum_{0<|m| \leqslant N\left(h_{n}\right)} c_{m}^{n} \Phi_{m, h}
$$

Then we have:

$$
\begin{gathered}
\psi_{n}=\frac{1}{\sqrt{2}} \sum_{m=1}^{N\left(h_{n}\right)}\left(c_{m}^{n}+c_{-m}^{n}\right) \varphi_{m, h_{n}}, \\
\sum_{M\left(h_{n}\right)<m \leqslant N\left(h_{n}\right)}\left|c_{m}^{n}+c_{-m}^{n}\right|^{2} \rightarrow 0, \\
\sum_{0<|m| \leqslant M\left(h_{n}\right)}\left|\omega_{n}-\lambda_{m, h_{n}}\right|^{2}\left|c_{m}^{n}\right|^{2} \rightarrow 0 .
\end{gathered}
$$


Proof. Relation (3.32) follows directly by taking the second component in (3.31) and by using (3.27).

From (3.21) and (3.32) it follows that

$$
h_{n}^{\theta}\left\|A_{0}^{\frac{1}{2}} \psi_{n}\right\|^{2}=\sum_{m=1}^{N\left(h_{n}\right)} h_{n}^{\theta} \lambda_{m, h_{n}}^{2}\left|c_{m}^{n}+c_{-m}^{n}\right|^{2} \rightarrow 0
$$

The above relation, (3.29) and (3.28) imply (3.33). Relations (3.30), (3.28) and (3.35) clearly imply that there exists a constant $\tilde{C}$ independent of $n$ such that

$$
h_{n}^{2 \theta} \sum_{m=1}^{M\left(h_{n}\right)} \lambda_{m, h_{n}}^{4}\left|c_{m}^{n}+c_{-m}^{n}\right|^{2} \leqslant \tilde{C} \varepsilon \sum_{m=1}^{M\left(h_{n}\right)} h_{n}^{\theta} \lambda_{m, h_{n}}^{2}\left|c_{m}^{n}+c_{-m}^{n}\right|^{2} \rightarrow 0 .
$$

On the other hand, using $\lambda_{m, h}=\lambda_{-m, h}$ and (3.27), a simple calculation shows that

$$
\left[\begin{array}{c}
0 \\
h_{n}^{\theta} A_{0 h_{n}} \psi_{n}
\end{array}\right]=\sum_{0<|m| \leqslant N\left(h_{n}\right)} \frac{h_{n}^{\theta}}{2} \lambda_{m, h_{n}}^{2}\left(c_{m}^{n}+c_{-m}^{n}\right) \Phi_{m, h_{n}}
$$

Relations (3.36) and (3.37) imply that

$$
\left[\begin{array}{c}
0 \\
h_{n}^{\theta} A_{0 h_{n}} \psi_{n}
\end{array}\right]-\sum_{M\left(h_{n}\right)<|m| \leqslant N\left(h_{n}\right)} \frac{h_{n}^{\theta}}{2} \lambda_{m, h_{n}}^{2}\left(c_{m}^{n}+c_{-m}^{n}\right) \Phi_{m, h_{n}} \rightarrow 0 .
$$

Moreover, by using (3.31) and the fact that $\Phi_{m, h}$ is an eigenvector of $A_{1 h}$ associated to the eigenvalue $i \lambda_{m, h}$, we have that

$$
i \omega_{n} z_{n}-A_{1 h_{n}} z_{n}=\sum_{0<|m| \leqslant N\left(h_{n}\right)} i\left(\omega_{n}-\lambda_{m, h_{n}}\right) c_{m}^{n} \Phi_{m, h_{n}} .
$$

The above relation, combined to (3.25) and (3.38) implies that

$$
\sum_{0<|m| \leqslant N\left(h_{n}\right)} i\left(\omega_{n}-\lambda_{m, h_{n}}\right) c_{m}^{n} \Phi_{m, h_{n}}+\sum_{M\left(h_{n}\right)<|m| \leqslant N\left(h_{n}\right)} \frac{h_{n}^{\theta}}{2} \lambda_{m, h_{n}}^{2}\left(c_{m}^{n}+c_{-m}^{n}\right) \Phi_{m, h_{n}} \rightarrow 0 .
$$

Since the family $\left(\Phi_{m, h_{n}}\right)$ is orthonormal, the above relation implies (3.34).

\section{Proof of the main Result}

The proof of Theorem 1.1 is based on a frequency domain characterization of the exponential stability of the semi-group $\mathbb{T}$ generated by an operator $A$. The basic characterization was given in Prüss [15], p. 852, and it implies that if the function $s \rightarrow\left\|(s I-A)^{-1}\right\|$ is bounded for $\operatorname{Re}(s)>0$, then $\mathbb{T}$ is exponentially stable. A little later and independently, an equivalent result was given by Huang [9]. Here we need a result which is closely related to the one just mentioned, without being an obvious consequence of it. This result concerns the uniform exponential stability of a sequence of semi-groups. In order to have a precise statement we give a definition.

Definition 4.1. Let $h^{*}>0$, let $\left(X_{h}\right)$ be a family of Hilbert spaces and let $\left(\mathbb{T}_{h}\right)$ be a family of semi-groups of linear operators such that, for all $h \in\left(0, h^{*}\right)$, we have that $\mathbb{T}_{h}$ is a linear $C^{0}$ semi-group in $X_{h}$. The family of semi-groups $\mathbb{T}_{h}$ is said uniformly exponentially stable if there exist constants $M, \alpha>0$ (independent of $\left.h \in\left(0, h^{*}\right)\right)$ such that

$$
\left\|\mathbb{T}_{h}(t)\right\|_{\mathcal{L}\left(X_{h}\right)} \leqslant M \mathrm{e}^{-\alpha t} \quad \forall t \geqslant 0
$$


Recall that a strongly continuous semigroup $\mathbb{T}$ is called a contraction semigroup if $\left\|\mathbb{T}_{t}\right\| \leqslant 1$ for all $t \geqslant 0$. We can now state the following uniform stability result, which is given in Liu and Zheng [12], p. 162, and which will be applied in the proof of Theorem 1.1.

Theorem 4.2. Let $\left(\mathbb{T}_{h}\right)$ be a family of contraction semigroups on the Hilbert space $X_{h}$ and let $\left(\widetilde{A}_{h}\right)$ be the corresponding infinitesimal generators. The family $\left(\mathbb{T}_{h}\right)$ is uniformly exponentially stable if and only if the two following conditions are satisfied:

(i) For all $h \in\left(0, h^{*}\right), i \mathbb{R}$ is contained in the resolvent set $\rho\left(\widetilde{A}_{h}\right)$ of $\widetilde{A}_{h}$.

(ii) $\sup _{h \in\left(0, h^{*}\right), \omega \in \mathbb{R}}\left\|\left(i \omega-\widetilde{A}_{h}\right)^{-1}\right\|_{\mathcal{L}\left(X_{h}\right)}<+\infty$.

We are now in a position to prove Theorem 1.1

Proof of Theorem 1.1. The proof is based on Theorem 4.2. Notice first that, for all $h \in\left(0, h^{*}\right)$, the family $\left(\mathrm{e}^{t \widetilde{A}_{h}}\right)$ forms a contraction semigroup. The fact that the family $\left(\widetilde{A}_{h}\right)$ satisfies condition (i) in Theorem 4.2 follows from Lemma 3.1. In order to show that the family $\left(\widetilde{A}_{h}\right)$ also satisfies condition (ii) in Theorem 4.2 we use a contradiction argument. Let $\left(h_{n}\right),\left(\omega_{n}\right)$, and $\left(z_{n}\right)=\left(\left[\begin{array}{l}\varphi_{n} \\ \psi_{n}\end{array}\right]\right)$ be three sequences satisfying $(3.19)$ and (3.20). We introduce the set

$$
\mathcal{F}=\left\{n \in \mathbb{N}\left|\exists m(n) \in \mathbb{Z}^{*},\right| m(n) \mid \leqslant M\left(h_{n}\right), \text { such that }\left|\omega_{n}-\lambda_{m(n), h_{n}}\right|<\frac{\gamma}{2}\right\},
$$

where $\gamma$ is defined in Proposition 2.2. We distinguish two cases.

First case. The set $\mathcal{F}$ is infinite. Then, for the sake of simplicity, we can suppose, without loss of generality, that $\mathcal{F}=\mathbb{N}$. Then, by reducing the value of $\gamma$ if needed, we can assume that for all $m \in \mathbb{Z}^{*}$ with $m \neq m(n)$ and $|m| \leqslant M\left(h_{n}\right)$, we have

$$
\left|\omega_{n}-\lambda_{m, h_{n}}\right| \geqslant \frac{\gamma}{2} .
$$

By using Lemma 2.1 we see that assumptions of Lemma 3.3 hold true. Thus, by using (3.34) we obtain that

$$
\sum_{\substack{0<|m| \leqslant M\left(h_{n}\right) \\ m \neq m(n)}}\left|c_{m}^{n}\right|^{2} \rightarrow 0 .
$$

Define now

$$
\widetilde{\psi_{n}}=\frac{1}{\sqrt{2}} c_{m(n)}^{n} \varphi_{m(n), h_{n}} .
$$

Relations (3.32), (3.33) and (4.1) show that

$$
\left\|\widetilde{\psi_{n}}-\psi_{n}\right\| \rightarrow 0
$$

Thus, since $\left(\left\|B_{0 h_{n}}^{*}\right\|\right)$ is bounded, we deduce that

$$
\left\|B_{0 h_{n}}^{*}\left(\psi_{n}-\widetilde{\psi_{n}}\right)\right\|_{U} \rightarrow 0
$$

The above relation and (3.21) imply that

$$
\left\|B_{0 h_{n}}^{*} \widetilde{\psi_{n}}\right\|_{U} \rightarrow 0
$$


But on the other hand, by using Proposition 2.2, we have that

$$
\left\|B_{0 h_{n}}^{*} \widetilde{\psi_{n}}\right\|_{U}=\frac{1}{\sqrt{2}}\left|c_{m(n)}^{n}\right|\left\|B_{0 h_{n}}^{*} \varphi_{m(n), h_{n}}\right\|_{U} \geqslant \frac{1}{\sqrt{2}} \beta\left|c_{m(n)}^{n}\right| .
$$

Gathering (4.2), (4.5) and (4.6), we obtain that $\widetilde{\psi_{n}} \rightarrow 0$ in $H$. By using then (4.3), we obtain that $\psi_{n} \rightarrow 0$ which contradicts (3.22).

Second case. The set $\mathcal{F}$ is finite. Then, we can assume, without loss of generality, that $\mathcal{F}$ is empty, i.e., that, for all $n \in \mathbb{N}$, we have that

$$
\left|\omega_{n}-\lambda_{m, h_{n}}\right| \geqslant \frac{\gamma}{2} \text { if } 0<|m| \leqslant M\left(h_{n}\right) .
$$

By using (3.34) and the above relation, we obtain that

$$
\sum_{0<|m| \leqslant M\left(h_{n}\right)}\left|c_{m}^{n}\right|^{2} \rightarrow 0 .
$$

The above relation, (3.32) and (3.33) imply that

$$
\psi_{n} \rightarrow 0 \text { in } H,
$$

which contradicts (3.22).

\section{Application to LQR optimization PRoBlems}

In this section, we are going to see how our uniform exponential stability result can be used to construct finite dimensional approximations of the Riccati operators appearing in the LQR analysis of vibrating systems. In order to make our statements precise, let us start by introducing the notation used in this section, and by recalling some classical results on Riccati operators in infinite dimensional spaces and on their approximation by finite dimensional Riccati operators.

Dealing with the optimal control of system (1.1)-(1.2), we first notice that this system can be easily written as a first order system,

$$
\dot{z}(t)=A z(t)+B u(t), \quad z(0)=z_{0},
$$

where the state $z(t)$ and the state space $X$ are defined by

$$
z(t)=\left[\begin{array}{l}
w(t) \\
\dot{w}(t)
\end{array}\right], \quad X=\mathcal{D}\left(A_{0}^{\frac{1}{2}}\right) \times H,
$$

whereas the operators $A, B$ and the initial state $z_{0}$ are given by

$$
\mathcal{D}(A)=\mathcal{D}\left(A_{0}\right) \times \mathcal{D}\left(A_{0}^{\frac{1}{2}}\right), \quad A=\left[\begin{array}{cc}
0 & I \\
-A_{0} & 0
\end{array}\right], \quad B=\left[\begin{array}{c}
0 \\
B_{0}
\end{array}\right], \quad z_{0}=\left[\begin{array}{c}
w_{0} \\
w_{1}
\end{array}\right] .
$$

The Hilbert space $X$ is equipped with the norm $\|\cdot\|_{X}$ defined by:

$$
\|z\|_{X}^{2}=\left\|z_{1}\right\|^{2}+\left\|z_{2}\right\|_{\frac{1}{2}}^{2}, \quad z=\left[\begin{array}{c}
z_{1} \\
z_{2}
\end{array}\right] .
$$

It is well-known that the above operator $A$ is skew-adjoint, and so, according to Stone's theorem, it generates a strongly continuous group of isometries in $X$. This fact implies that, if $u \in L^{2}(0, \infty, U)$ and $z_{0} \in X$, then (5.1) admits a unique solution $z \in C(0, \infty ; X)$. 
We associate to (5.1) the cost functional

$$
J\left(w_{0}, w_{1} ; u\right)=\int_{0}^{\infty}\left(\|u(t)\|_{U}^{2}+\|z(t)\|_{X}^{2}\right) \mathrm{d} t .
$$

If we assume that the system (5.1) is optimizable, i.e., that, for all $\left(w_{0}, w_{1}\right) \in X$, there exists an input $u \in$ $L^{2}(0, \infty ; U)$ such that $J\left(w_{0} ; w_{1} ; u\right)<\infty$ then (see, for instance, Curtain and Zwart [4], p. 294) there exists a self-adjoint and nonnegative operator $P \in \mathcal{L}(X)$ such that

$$
\min _{u \in L^{2}(0 ; \infty, U)} J\left(w_{0}, w_{1} ; u\right)=\left\langle\left[\begin{array}{l}
w_{0} \\
w_{1}
\end{array}\right], P\left[\begin{array}{l}
w_{0} \\
w_{1}
\end{array}\right]\right\rangle_{X} .
$$

Furthermore, the optimal control $u_{\text {opt }}$ is given by the feedback law

$$
u_{\mathrm{opt}}(t)=-B^{*} P S(t)\left[\begin{array}{l}
w_{0} \\
w_{1}
\end{array}\right],
$$

where $S$ is the strongly continuous semigroup generated by $A-B B^{*} P$. The operator $P$, called the Riccati operator, satisfies the algebraic Riccati equation

$$
\left\langle A z_{1}, P z_{2}\right\rangle+\left\langle P z_{1}, A z_{2}\right\rangle-\left\langle B^{*} P z_{2}, B^{*} P z_{1}\right\rangle+\left\langle z_{2}, z_{1}\right\rangle=0 \quad \forall z_{1}, z_{2} \in \mathcal{D}(A)
$$

Moreover, if we assume that the system (5.1) is exponentially stabilizable (i.e., that there exists $K \in \mathcal{L}(X, U)$ such that $A+B K$ generates an exponentially stable semigroup), then $P$ is the unique nonnegative solution of (5.3) (see, for instance, [4], p. 299).

As it has been already said, our goal in this section is to generate finite dimensional approximations of the Riccati operator $P$ and of the gain operator $-B^{*} P$. More precisely, we want to construct two sequences of subspaces $\left(X_{h}\right) \subset X,\left(U_{h}\right) \subset U$, and the approximate control systems

$$
\dot{z}_{h}(t)=A_{h} z_{h}(t)+B_{h} u_{h}(t), \quad z_{h}(0)=z_{0 h}
$$

where $A_{h} \in \mathcal{L}\left(X_{h}\right), B_{h} \in \mathcal{L}\left(U_{h}, X_{h}\right)$ and such that the two following conditions hold:

- For any positive $h$, with $h$ small enough, the system (5.4) is exponentially stabilizable.

- The corresponding sequence $\left(P_{h}\right)$ of approximate Riccati operators strongly converges to the Riccati operator $P$, i.e., if $\sigma_{h}$ denotes the orthogonal projection from $X$ onto $X_{h}$, we have

$$
\lim _{h \rightarrow 0} P_{h} \sigma_{h} z=P z \quad \forall z \in X
$$

Several works (see, for instance, Banks and Kunisch [1], Gibson [6], Gibson and Adamian [7], Kappel and Salamon [11]) contain sufficient conditions for the strong convergence of $P_{h}$ to $P$. In particular, the following result holds.

Theorem 5.1. Let $\rho_{h}$ the orthogonal projection from $U$ onto $U_{h}$ and let $S_{h}$ be the semigroup of linear operators in $X_{h}$ generated by $A_{h}$. We assume that:

(1) The system (5.1) is exponentially stabilizable.

(2) $S_{h}(t) \sigma_{h} z \rightarrow S(t) z$ and $S_{h}^{*}(t) \sigma_{h} z \rightarrow S^{*}(t) z$ for all $z \in X$, with uniform convergence with respect to $t$ in bounded subsets of $[0, \infty)$.

(3) $B_{h} \rho_{h} u \rightarrow B u$ in $X$, for all $u \in U$ and $B_{h}^{*} \sigma_{h} z \rightarrow B^{*} z$ for all $z \in X$.

(4) The family of pairs $\left(A_{h}, B_{h}\right)$ is uniformly stabilizable, i.e., there exists a sequence of operators $\left(K_{h}\right)$ such that

$$
\left\|K_{h}\right\|_{\mathcal{L}\left(X_{h}\right)} \leqslant M_{0} \quad \forall h \in\left(0, h^{*}\right),
$$


and the semigroup $S_{A_{h}-B_{h} K_{h}}$ generated by $A_{h}-B_{h} K_{h}$ satisfies

$$
\left\|S_{A_{h}-B_{h} K_{h}}(t)\right\|_{\mathcal{L}\left(X_{h}\right)} \leqslant M_{1} \mathrm{e}^{-\alpha_{1} t} \quad \forall t \geqslant 0
$$

for some positive constants $M_{0}, M_{1}$ and $\alpha_{1}$ (independent of $h$ ).

Then, the sequence $\left(P_{h}\right)$ strongly converges to $P$, in the sense of (5.5).

The main difficulty in applying the above result to the approximation of systems governed by hyperbolic partial differential equations (like our examples in Sect. 6) consists in checking assumption (4) in Theorem 5.1. Indeed, as it has been already said in the introduction, the usual finite element approximation naturally leads to numerical schemes that do not, in general, satisfy the uniform exponential decay property (see for instance [2], for the problem of the approximation of the wave equation with boundary control). The introduction of a numerical viscosity in the numerical scheme, as seen in Section 4, ensures the uniform exponential stability and allows us to use Theorem 5.1 to derive the main result of this section, concerning the convergence of the Riccati operators. Before stating this result, let us introduce some further useful notations.

The second order system with numerical damping (1.14)-(1.15) can be written as a first order one of the form (5.4) by setting

and

$$
X_{h}=V_{h} \times V_{h}, \quad z_{h}(t)=\left[\begin{array}{c}
w_{h}(t) \\
\dot{w}_{h}(t)
\end{array}\right], \quad z_{0 h}=\left[\begin{array}{l}
w_{0 h} \\
w_{1 h}
\end{array}\right]
$$

$$
A_{h}=\left[\begin{array}{cc}
0 & I \\
-A_{0 h} & -h^{\theta} A_{0 h}
\end{array}\right], \quad B_{h}=\left[\begin{array}{c}
0 \\
B_{0 h}
\end{array}\right] .
$$

The space $X_{h}$ is endowed with the inner product of $X$ :

$$
\left\langle\left(\varphi_{h}, \psi_{h}\right),\left(\widetilde{\varphi}_{h}, \widetilde{\psi}_{h}\right)\right\rangle_{X_{h}}=\left\langle A_{0 h}^{\frac{1}{2}} \varphi_{h}, A_{0 h}^{\frac{1}{2}} \widetilde{\varphi}_{h}\right\rangle+\left\langle\psi_{h}, \widetilde{\psi}_{h}\right\rangle .
$$

Then, the following result holds.

Theorem 5.2. Suppose that the operators $A_{0}, B_{0}$, the spaces $H, U$ and the approximating families $\left(V_{h}\right)$ and $\left(U_{h}\right)$ satisfy the assumptions in Theorem 1.1. Then the algebraic Riccati equation

$$
A_{h}^{*} P_{h}+P_{h} A-P_{h} B_{h} B_{h}^{*} P_{h}+I=0
$$

(resp. the equation (5.3)) admits a unique nonnegative solution $P_{h}$ (resp. $\left.P\right)$. Moreover, the sequence $\left(P_{h}\right)$ strongly converges to $P$, in the sense of (5.5).

Proof. It suffices to show that our hypothesis imply that $A_{0}, B_{0}$, the spaces $H, U$ and the approximating families $\left(V_{h}\right)$ and $\left(U_{h}\right)$ satisfy assumptions $(1-4)$ in Theorem 5.1.

Due to (1.12), (1.13), we can apply Theorem 1 in [3] to get that the system (5.1) is exponentially stabilizable (a stabilizing feedback operator is $K=-B^{*}$ ). We have thus shown that assumption (1) in Theorem 5.1 is satisfied.

We next show that assumption (3) in Theorem 5.1 is also satisfied. Since $B_{h} \rho_{h} u=\left[\begin{array}{c}0 \\ \widetilde{\pi}_{h} B_{0} \rho_{h} u\end{array}\right]$ and $B u=$ $\left[\begin{array}{c}0 \\ B_{0} u\end{array}\right]$, the first assertion of assumption (3) (i.e. $B_{h} \rho_{h} u \rightarrow B u$ in $X$ for all $u \in U$ ) holds if and only if

$$
\left\|\tilde{\pi}_{h} B_{0} \rho_{h} u-B_{0} u\right\| \rightarrow 0 \quad \forall u \in U .
$$

Writing that

$$
\begin{aligned}
\left\|\widetilde{\pi}_{h} B_{0} \rho_{h} u-B_{0} u\right\| & \leqslant\left\|\widetilde{\pi}_{h}\left(B_{0} \rho_{h} u-B_{0} u\right)\right\|+\left\|\widetilde{\pi}_{h}\left(B_{0} u\right)-B_{0} u\right\| \\
& \leqslant\left\|B_{0} \rho_{h} u-B_{0} u\right\|+\left\|\widetilde{\pi}_{h}\left(B_{0} u\right)-B_{0} u\right\|
\end{aligned}
$$


and using the properties (1.9) and (1.11) satisfied by the approximation space $V_{h}$, and the fact that $B_{0} \in$ $\mathcal{L}(U, H)$, we immediately get (5.8). We turn now to the second assumption appearing in assumption (3), which reads:

$$
\left\|B_{h}^{*} \sigma_{h} z-B^{*} z\right\|_{U} \rightarrow 0 \quad \forall z \in X .
$$

It can be easily checked that

$$
\left\|B_{h}^{*} \sigma_{h} z-B^{*} z\right\|_{U}=\left\|\rho_{h} B_{0}^{*} \psi_{h}-B_{0}^{*} \psi\right\|_{U},
$$

where we have set $z=\left[\begin{array}{l}\varphi \\ \psi\end{array}\right]$ and $\sigma_{h} z=\left[\begin{array}{l}\varphi_{h} \\ \psi_{h}\end{array}\right]=\left[\begin{array}{c}\pi_{h} \varphi \\ \widetilde{\pi}_{h} \psi\end{array}\right]$. Hence

$$
\begin{aligned}
\left\|B_{h}^{*} \sigma_{h} z-B^{*} z\right\|_{U} & \leqslant\left\|\rho_{h} B_{0}^{*}\left(\psi_{h}-\psi\right)\right\|_{U}+\left\|\rho_{h}\left(B_{0}^{*} \psi\right)-B_{0}^{*} \psi\right\|_{U} \\
& \leqslant\left\|B_{0}^{*}\right\| \cdot\left\|\psi-\psi_{h}\right\|+\left\|\rho_{h}\left(B_{0}^{*} \psi\right)-B_{0}^{*} \psi\right\|_{U} .
\end{aligned}
$$

Relation (5.9) follows then immediately from (1.11) and (1.9).

Our main result Theorem 1.1 shows that assumption (4) holds (with $K_{h}=-B_{h}^{*}$ ).

To achieve the proof, it remains to check that assumption (2) is satisfied. This result is given by Proposition 5.3 below.

Proposition 5.3. Suppose that the operators $A_{0}, B_{0}$, the spaces $H, U$ and the approximating families $\left(V_{h}\right)$ and $\left(U_{h}\right)$ satisfy the assumptions in Theorem 1.1. Let $S, S_{h}$ and $S_{h}^{*}$ denote the semigroups generated respectively by $A, A_{h}$ and $A_{h}^{*}\left(A, A_{h}\right.$ are defined respectively by (5.2), (5.6)). Moreover, let $\sigma_{h}$ be the orthogonal projection from $X$ onto $X_{h}$. Then, for all $z \in X$ and for all $t \geqslant 0$ we have:

(i) $S_{h}(t) \sigma_{h} z \rightarrow S(t) z$;

(ii) $S_{h}^{*}(t) \sigma_{h} z \rightarrow S^{*}(t) z$

with uniform convergence with respect to $t$ in bounded subsets of $[0, \infty)$.

Proof.

(i) First, it is clear that $\left\|S_{h}(t)\right\| \leqslant 1,\|S(t)\|=1$, for all $t \geqslant 0$. Consequently, by using a version of the Trotter-Kato Theorem (see, for instance, the remark following Th. 4.2 in [14], p. 86), it suffices to prove that

$$
\left(I-A_{h}\right)^{-1} F_{h} \longrightarrow(I-A)^{-1} F \quad \forall F=\left[\begin{array}{c}
f \\
g
\end{array}\right] \in \mathcal{D}\left(A_{0}^{\frac{1}{2}}\right) \times H,
$$

where $F_{h}=\left[\begin{array}{l}f_{h} \\ g_{h}\end{array}\right]=\left[\begin{array}{c}\pi_{h} f \\ \widetilde{\pi}_{h} g\end{array}\right] \in V_{h} \times V_{h}$. Note that, by construction, we have

$$
\left\|F_{h}-F\right\|_{X}^{2}=\left\|f_{h}-f\right\|_{\frac{1}{2}}^{2}+\left\|g_{h}-g\right\|^{2} \rightarrow 0 .
$$

In order to prove (5.10) we notice that $(I-A)^{-1}\left[\begin{array}{l}f \\ g\end{array}\right]=\left[\begin{array}{l}\varphi \\ \psi\end{array}\right]$ if and only if

$$
\left\{\begin{aligned}
A_{0} \varphi+\varphi & =f+g \\
\psi & =\varphi-f
\end{aligned}\right.
$$

and similarly, that $\left(I-A_{h}\right)^{-1}\left[\begin{array}{l}f_{h} \\ g_{h}\end{array}\right]=\left[\begin{array}{l}\varphi_{h} \\ \psi_{h}\end{array}\right]$ if and only if

$$
\left\{\begin{aligned}
A_{0 h} \varphi_{h}+\varphi_{h}+h^{\theta} A_{0 h} \varphi_{h} & =f_{h}+g_{h}+h^{\theta} A_{0 h} f_{h} \\
\psi_{h} & =\varphi_{h}-f_{h} .
\end{aligned}\right.
$$


Consequently, in order to prove (5.10), it suffices to show that the error $e_{h}=\varphi_{h}-\varphi$ converges to 0 in $\mathcal{D}\left(A_{0}^{\frac{1}{2}}\right)$. By taking the inner product in $H$ of the first relation in (5.12) (resp. in (5.13)) by $\zeta \in \mathcal{D}\left(A_{0}^{\frac{1}{2}}\right)$ (resp. by $\left.\zeta_{h} \in V_{h}\right)$ we obtain that $\varphi \in \mathcal{D}\left(A_{0}^{\frac{1}{2}}\right)$ (resp. $\left.\varphi_{h} \in V_{h}\right)$ satisfies:

$$
a_{0}(\varphi, \zeta)=\langle f+g, \zeta\rangle
$$

and

$$
a_{0}\left(\varphi_{h}, \zeta_{h}\right)+h^{\theta}\left\langle A_{0}^{\frac{1}{2}} \varphi_{h}, A_{0}^{\frac{1}{2}} \zeta_{h}\right\rangle=\left\langle f_{h}+g_{h}, \zeta_{h}\right\rangle+h^{\theta}\left\langle A_{0}^{\frac{1}{2}} f_{h}, A_{0}^{\frac{1}{2}} \zeta_{h}\right\rangle
$$

where $a_{0}(\cdot, \cdot)$ denotes the inner product of $\mathcal{D}\left(A_{0}^{\frac{1}{2}}\right)$ defined by

$$
a_{0}(\varphi, \zeta)=\left\langle A_{0}^{\frac{1}{2}} \varphi, A_{0}^{\frac{1}{2}} \zeta\right\rangle+\langle\varphi, \zeta\rangle
$$

Taking $\zeta_{h}=\varphi_{h}$ in (5.15), we get that $\varphi_{h}$ is bounded in $\mathcal{D}\left(A_{0}^{\frac{1}{2}}\right)$. On the other hand, since $V_{h} \subset \mathcal{D}\left(A_{0}^{\frac{1}{2}}\right)$, we can take $\zeta=\zeta_{h}$ in (5.14) and subtracting from (5.15), we see that the error $e_{h}=\varphi_{h}-\varphi$ satisfies, for all test function $\zeta_{h} \in V_{h}$, the relation:

$$
a_{0}\left(e_{h}, \zeta_{h}\right)=h^{\theta}\left\langle A_{0}^{\frac{1}{2}} f_{h}-A_{0}^{\frac{1}{2}} \varphi_{h}, A_{0}^{\frac{1}{2}} \zeta_{h}\right\rangle+\left\langle\left(f_{h}-f\right)+\left(g_{h}-g\right), \zeta_{h}\right\rangle .
$$

For all $\widetilde{\varphi}_{h} \in V_{h}$, we can write that

$$
a_{0}\left(e_{h}, e_{h}\right)=a_{0}\left(e_{h}, \varphi_{h}-\varphi\right)=a_{0}\left(e_{h}, \varphi_{h}-\widetilde{\varphi}_{h}\right)+a_{0}\left(e_{h}, \widetilde{\varphi}_{h}-\varphi\right) .
$$

Thanks to (5.16) used with the test function $\zeta_{h}=\varphi_{h}-\widetilde{\varphi}_{h} \in V_{h}$ and to the boundedness of $\left(\varphi_{h}\right)$ in $\mathcal{D}\left(A_{0}^{\frac{1}{2}}\right)$, the first term of the right-hand side of (5.17) satisfies:

$$
\left|a_{0}\left(e_{h}, \varphi_{h}-\widetilde{\varphi}_{h}\right)\right| \leqslant \mu(h)\left\|\varphi_{h}-\widetilde{\varphi}_{h}\right\|_{\frac{1}{2}},
$$

where $\mu(h)=C\left(h^{\theta}+\left\|f_{h}-f\right\|+\left\|g_{h}-g\right\|\right)$ (here and in the rest of the proof, $C$ denotes a constant independent of $h)$. By (5.11), we have

$$
\lim _{h \rightarrow 0} \mu(h)=0 .
$$

Now, we can write that

$$
\begin{aligned}
\left|a_{0}\left(e_{h}, \varphi_{h}-\widetilde{\varphi}_{h}\right)\right| & \leqslant \mu(h)\left\|\varphi_{h}-\widetilde{\varphi}_{h}\right\|_{\frac{1}{2}} \\
& \leqslant \mu(h)\left(\left\|e_{h}\right\|_{\frac{1}{2}}+\left\|\varphi-\widetilde{\varphi}_{h}\right\|_{\frac{1}{2}}\right) \\
& \leqslant \frac{1}{2 \eta} \mu(h)^{2}+\eta\left(\left\|e_{h}\right\|_{\frac{1}{2}}^{2}+\left\|\varphi-\widetilde{\varphi}_{h}\right\|_{\frac{1}{2}}^{2}\right),
\end{aligned}
$$

where the fixed constant $\eta>0$ will be chosen later.

Concerning the second term of the right-hand side of (5.17), we have by Cauchy-Schwarz inequality:

$$
\begin{aligned}
\left|a_{0}\left(e_{h}, \tilde{\varphi}_{h}-\varphi\right)\right| & \leqslant C\left\|e_{h}\right\|_{\frac{1}{2}}\left\|\tilde{\varphi}_{h}-\varphi\right\|_{\frac{1}{2}} \\
& \leqslant C\left(\frac{\eta}{2}\left\|e_{h}\right\|_{\frac{1}{2}}^{2}+\frac{1}{2 \eta}\left\|\tilde{\varphi}_{h}-\varphi\right\|_{\frac{1}{2}}^{2}\right) .
\end{aligned}
$$

Thus, we have shown that:

$$
\left\|e_{h}\right\|_{\frac{1}{2}}^{2} \leqslant a_{0}\left(e_{h}, e_{h}\right) \leqslant \eta\left(1+\frac{C}{2}\right)\left\|e_{h}\right\|_{\frac{1}{2}}^{2}+\frac{1}{2 \eta} \mu(h)^{2}+\left(\eta+\frac{C}{2 \eta}\right) \inf _{\tilde{\varphi}_{h} \in V_{h}}\left\|\varphi-\tilde{\varphi}_{h}\right\|_{\frac{1}{2}}^{2} .
$$


As a consequence, provided $\eta$ is chosen small enough, this relation shows that $\varphi_{h}$ converges to $\varphi$ in $\mathcal{D}\left(A_{0}^{\frac{1}{2}}\right)$, since $\inf _{\tilde{\varphi}_{h} \in V_{h}}\left\|\varphi-\tilde{\varphi}_{h}\right\|_{\frac{1}{2}}=\left\|\varphi-\pi_{h} \varphi\right\|_{\frac{1}{2}}$ converges to 0 by assumption (1.7). Thus, part (i) of the proposition is proved.

(ii) To prove the convergence of the semigroup generated by the adjoint $A_{h}^{*}$ of $A_{h}$, we first notice from a straightforward computation that

$$
A_{h}^{*}=\left[\begin{array}{ll}
0 & -I \\
A_{0 h} & -h^{\theta} A_{0 h}
\end{array}\right] .
$$

As a consequence, $\left(I-A_{h}^{*}\right)^{-1}\left[\begin{array}{l}f_{h} \\ g_{h}\end{array}\right]=\left[\begin{array}{l}\varphi_{h} \\ \psi_{h}\end{array}\right]$ if and only if

$$
\left\{\begin{aligned}
A_{0 h} \varphi_{h}+\varphi_{h}+h^{2 \theta} A_{0 h} \varphi_{h} & =f_{h}-g_{h}+h^{2 \theta} A_{0 h} f \\
\psi_{h} & =f_{h}-\varphi_{h}
\end{aligned}\right.
$$

Comparing the above system to the one obtained in (5.13) for the resolvent $\left(I-A_{h}\right)^{-1}$ of $A_{h}$, we see that the convergence of $S_{h}^{*}(t) \sigma_{h} z$ follows immediately from part $(i)$ of the proof.

\section{Applichtion to a nON homogeneous string EQUATION}

In this section we tackle the vibrations of an non homogeneous string under the action of a feedback control acting in a subinterval $[a, b]$ of $[0,1]$. More precisely we consider the following initial and boundary value problem:

$$
\begin{array}{cc}
\frac{\partial^{2} w}{\partial t^{2}}-\frac{\partial}{\partial x}\left[p(x) \frac{\partial w}{\partial x}\right]+\frac{\partial w}{\partial t} \chi_{[a, b]}=0 & \forall(x, t) \in(0,1) \times(0, \infty), \\
w(0, t)=w(1, t)=0 \quad \forall t \geqslant 0, & \forall x \in(0,1),
\end{array}
$$

where $\chi_{[a, b]}$ is the characteristic function of $[a, b]$ and $p \in C^{1}([0,1])$ is such that $p(x)>0$ for all $x \in[0,1]$. Problem (6.1)-(6.3) can be written in the form (1.3)-(1.4), if we introduce the appropriate spaces and operators. We start by defining $H=L^{2}(0,1)$ and the operator $A_{0}: \mathcal{D}\left(A_{0}\right) \rightarrow H$ by

$$
\begin{aligned}
\mathcal{D}\left(A_{0}\right) & =\left\{\varphi \in H_{0}^{1}(0,1) \mid p \frac{\mathrm{d} \varphi}{\mathrm{d} x} \in H^{1}(0,1)\right\}, \\
A_{0} \phi & =-\frac{\mathrm{d}}{\mathrm{d} x}\left[p \frac{\mathrm{d} \varphi}{\mathrm{d} x}\right] \quad \forall \varphi \in \mathcal{D}\left(A_{0}\right) .
\end{aligned}
$$

We then define the input space $U$ by $U=L^{2}(a, b)$ and the input operator $B_{0} \in \mathcal{L}(U, H)$ by

$$
B_{0} u=\widetilde{u} \chi_{[a, b]} \quad \forall u \in U,
$$

where we have denoted by $\widetilde{u}$ an extension of $u$ to an element of $L^{2}(0,1)$. Notice that

$$
B_{0}^{*} \varphi=\varphi_{\mid[a, b]} \quad \forall \varphi \in H .
$$

If we assume that $p \in C^{1}([0,1])$ and that $p(x)>0$ for all $x \in[0,1]$, then it can be easily checked that $A_{0}$ is self-adjoint, positive and boundedly invertible. A simple calculation shows that

$$
\mathcal{D}\left(A_{0}^{\frac{1}{2}}\right)=H_{0}^{1}(0,1),
$$


with the corresponding norm

$$
\|\varphi\|_{\frac{1}{2}}^{2}=\int_{0}^{1} p(x)\left|\frac{\mathrm{d} \varphi}{\mathrm{d} x}(x)\right|^{2} \mathrm{~d} x .
$$

Moreover, by using the definitions in Section 5, we can write (6.1)-(6.3) as a first order system with state space $X=H_{0}^{1}(0,1) \times L^{2}(0,1)$ and with input space $U=L^{2}(a, b)$. Let us first notice that the following result holds.

Proposition 6.1. Suppose that $p \in C^{1}([0,1])$ is such that $p(x)>0$ for all $x \in[0,1]$. Then $\mathcal{D}\left(A_{0}\right)=H^{2}(\Omega) \cap$ $H_{0}^{1}(\Omega)$ and the eigenvalues of the operator $A_{0}$ defined in (6.4)-(6.5), are simple. If we denote by $\left(\lambda_{n}\right)_{n \geqslant 1}$ the increasing sequence formed by the eigenvalues of $A_{0}^{\frac{1}{2}}$ then there exists a constant $\gamma_{0}>0$ such that

$$
\lambda_{n+1}-\lambda_{n} \geqslant \gamma_{0}>0 \quad \forall n \geqslant 1 .
$$

Moreover, if we denote by $\left(\varphi_{n}\right)$ an orthonormal basis of $H$ formed by eigenvectors of $A_{0}$ then there exists a constant $\beta_{0}>0$ such that

$$
\left\|B_{0}^{*} \varphi_{n}\right\| \geqslant \beta_{0} \quad \forall n \in \mathbb{N}
$$

Proof. The conclusion follows from our assumptions on $p$ combined to classical asymptotic estimates for eigenvalues and eigenfunctions of second order differential equations (see, for instance, [13], Chap. 2).

In order to construct the spaces $V_{h}$ and $U_{h}$ we use $P 1$ finite elements. More precisely, if $h>0$ and $N(h) \in \mathbb{N}$ are such that $h=\frac{1}{N(h)+1}$ we define the points

$$
x_{j}=j h, j=1, \ldots, N(h) .
$$

The space $V_{h}$ is the linear span of the family of hat functions

$$
e_{j}(x)=\left[1-\frac{\left|x-x_{j}\right|}{h}\right]^{+}, \quad \forall j \in 1, \ldots, N(h) .
$$

The space $U_{h}$ is defined as the space formed by all restrictions to $[a, b]$ of functions in $V_{h}$. Moreover, we define the operators $A_{0 h}$ and $B_{0 h}$ by (1.5) and (1.6).

Proposition 6.2. The operators $A_{0}, B_{0}$ and the spaces $V_{h}, U_{h}$ defined above satisfy conditions (1.7), (1.10) with $\theta=1$.

Proof. Since $p \in C^{1}([0,1])$ is bounded away from zero, it can be easily checked that that graph norm of $A_{0}$ is equivalent to the norm in $H^{2}(0,1)$, so estimates (1.7)-(1.9) (with $\theta=1$ ) follow by Theorem 1.3 in [17], p. 45. By using (6.6) and Theorem 1.3 in [17], p. 45, we obtain that condition (1.10) is also satisfied with $\theta=1$.

Remark 6.3. By using the techniques in [5] the results in Proposition 6.1 can be extended for a function $p$ which is only piecewise $C^{1}$. Moreover, by slightly modifying the mesh used in our discretization procedure, one can show that the result in Proposition 6.2 still holds for $p$ piecewise $C^{1}$.

Proposition 6.1 and in Proposition 6.2 imply that our main results (Ths. 1.1 and 5.2) can be applied to system (6.1)-(6.3). In particular, Theorem 1.1 shows that the solutions $w_{h}$ of

$$
\begin{gathered}
\left\langle\ddot{w}_{h}, \varphi_{h}\right\rangle+\left\langle p(x) \frac{\partial w_{h}}{\partial x}, \frac{\mathrm{d} \varphi_{h}}{\mathrm{~d} x}\right\rangle+\int_{a}^{b} \dot{w}_{h} \varphi_{h} \mathrm{~d} x+h\left\langle p(x) \frac{\partial \dot{w}_{h}}{\partial x}, \frac{\mathrm{d} \varphi_{h}}{\mathrm{~d} x}\right\rangle=0 \quad \forall \varphi_{h} \in V_{h} \\
w_{h}(x, 0)=w_{0 h}(x), \quad \frac{\partial w_{h}}{\partial t}(x, 0)=w_{1 h}(x) \quad \forall x \in(0,1),
\end{gathered}
$$

are uniformly exponentially stable in $H_{0}^{1}(0,1) \times L^{2}(0,1)$.

Remark 6.4. In the case of a constant function $p$ in the above system, Tcheugoué Tébou and Zuazua in [18] showed a similar uniform exponential stability result with a numerical viscosity proportional to $h^{2}$ instead of $h$. 


\section{A 2-D Plate EQUATion in THE SQUARE}

In this section we tackle the vibrations of an elastic square plate under the action of a feedback damping supported in a horizontal strip. More precisely, let $\Omega$ be the square $\Omega=(0, \pi) \times(0, \pi)$ and let $0<a<b<\pi$. We consider the following initial and boundary value problem:

$$
\begin{gathered}
\frac{\partial^{2} w}{\partial t^{2}}+\Delta^{2} w+\chi_{[0, \pi] \times[a, b]} \frac{\partial w}{\partial t}=0, \quad x \in \Omega, t \geqslant 0, \\
w(t)=\Delta w(t)=0, x \in \partial \Omega, t \geqslant 0, \\
w(x, 0)=w_{0}(x), \quad \frac{\partial w}{\partial t}(x, 0)=w_{1}(x) \quad \forall x \in \Omega,
\end{gathered}
$$

where $\chi_{[0, \pi] \times[a, b]}$ is the characteristic function of $[0, \pi] \times[a, b]$. Equations (7.9)-(7.11) can be written in the form (1.3)-(1.4) if we introduce the following notation:

$$
\begin{gathered}
H=L^{2}(\Omega), \quad \mathcal{D}\left(A_{0}\right)=\left\{\varphi \in H^{4}(\Omega) \cap H_{0}^{1}(\Omega) \mid \Delta \varphi=0 \text { on } \partial \Omega\right\}, \\
A_{0}: \mathcal{D}\left(A_{0}\right) \rightarrow H, \quad A_{0} \varphi=\Delta^{2} \varphi \quad \forall \varphi \in \mathcal{D}\left(A_{0}\right) .
\end{gathered}
$$

It can be easily checked that $A_{0}$ is self-adjoint, positive and boundedly invertible. A simple calculation shows that

with the corresponding norm

$$
\mathcal{D}\left(A_{0}^{\frac{1}{2}}\right)=H^{2}(\Omega) \cap H_{0}^{1}(\Omega),
$$

$$
\|\varphi\|_{\frac{1}{2}}^{2}=\int_{\Omega}|\Delta \varphi(x)|^{2} \mathrm{~d} x
$$

The eigenvalues of $A_{0}^{\frac{1}{2}}$ are

$$
\widetilde{\lambda}_{p, q}=p^{2}+q^{2} \quad \forall p, q \in \mathbb{N}^{*} .
$$

We define the input space by $U=L^{2}((0, \pi) \times(a, b))$ and the input operator $B_{0} \in \mathcal{L}(U, H)$ by

$$
B_{0} u=\widetilde{u} \chi_{[0, \pi] \times[a, b]} \quad \forall u \in U,
$$

where we have denoted by $\widetilde{u}$ an extension of $u$ to an element of $L^{2}(\Omega)$.

Clearly, $A_{0}$ does not satisfy the assumptions of Theorem 1.1. Thus, any approximation $A_{0 h}$ of $A_{0}$ will not satisfy the uniform gap condition (2.8). Nevertheless, by adapting the proof of Theorem 1.1, we will see that the numerical viscosity method still yields uniformly stable discretizations of the closed loop system

$$
\begin{gathered}
\ddot{w}+A_{0} w+B_{0} B_{0}^{*} \dot{w}=0 \\
w(0)=w_{0}, \quad \dot{w}(0)=w_{1} .
\end{gathered}
$$

The discretized version of system (7.15)-(7.16) is obtained here by using a finite difference semi-discretization. This illustrates that the general approach presented in this paper can be adapted to deal with different kinds of approximations.

We detail now the finite difference discretization procedure. Given $\widetilde{N} \in \mathbb{N}$, we set

$$
h=\frac{\pi}{\widetilde{N}+1} .
$$

For the sake of simplicity, we can assume without loss of generality that there exist two integers $a(h) \geqslant 1$ and $b(h) \leqslant \widetilde{N}$ such that

$$
a=a(h) h, \quad b=b(h) h .
$$


Set

$$
V_{h}=\mathbb{R}^{\left(\tilde{N}^{2}\right)} \quad U_{h}=\mathbb{R}^{\tilde{N} \times(b(h)-a(h)+1)}
$$

and let $w_{j, k}$ denote for all $j, k \in\{0, \widetilde{N}+1\}$ the approximation of the solution $w$ of the system (7.9)-(7.11) at the point $x_{j, k}=(j h, k h)$. We use the standard finite difference approximation of the laplacian

$$
\Delta w(j h, k h) \approx \frac{1}{h^{2}}\left(w_{j+1, k}+w_{j-1, k}+w_{j, k+1}+w_{j, k-1}-4 w_{j, k}\right) \quad \forall j, k \in\{1, \widetilde{N}\} .
$$

In order to satisfy the boundary conditions (7.10) we set

$$
\begin{gathered}
w_{0, k}=w_{k, 0}=w_{\tilde{N}+1, k}=w_{k, \tilde{N}+1}=0 \quad \forall k \in\{0, \ldots, \widetilde{N}+1\}, \\
w_{-1, k}=-w_{1, k}, w_{\widetilde{N}+2, k}=-w_{\widetilde{N}, k}, w_{k,-1}=-w_{k, 1}, w_{k, \widetilde{N}+2}=-w_{k, \widetilde{N}} \quad \forall k \in\{0, \ldots, \widetilde{N}+1\} .
\end{gathered}
$$

Let $w_{h} \in V_{h}$ be the vector whose components are the $w_{j, k}$ for $1 \leqslant j, k \leqslant \widetilde{N}$. We define the matrix $A_{0 h}$ representing the discretization of the bilaplacian with hinged boundary conditions via its square root $A_{0 h}^{\frac{1}{2}}$ given by

$$
\left(A_{0 h}^{\frac{1}{2}} w_{h}\right)_{j, k}=-\frac{1}{h^{2}}\left(w_{j+1, k}+w_{j-1, k}+w_{j, k+1}+w_{j, k-1}-4 w_{j, k}\right)
$$

for all $1 \leqslant j, k \leqslant \tilde{N}$. The finite-difference approximation $B_{0 h}^{*} \in \mathcal{L}\left(V_{h}, U_{h}\right)$ of $B_{0}^{*}$ is defined by

$$
\left(B_{0 h}^{*} w_{h}\right)_{j, k}=w_{j, k} \quad \text { for all } 1 \leqslant j \leqslant \widetilde{N}, a(h) \leqslant k \leqslant b(h)
$$

We use semi-discretization of (7.15)-(7.16) of type (1.14) with $\theta=2$, namely

$$
\begin{gathered}
\ddot{w}_{j, k}+\left(A_{0 h} w_{h}\right)_{j, k}+h^{2}\left(A_{0 h} \dot{w}_{h}\right)_{j, k}+\left(B_{0 h} B_{0 h}^{*} \dot{w}_{h}\right)_{j, k}=0, \quad 1 \leqslant j, k \leqslant \tilde{N}, \quad t \geqslant 0, \\
w_{j, k}=\left(A_{0 h}^{\frac{1}{2}} w_{h}\right)_{j, k}=0, \quad j, k \in\{0, \widetilde{N}+1\}, \quad t \geqslant 0 \\
w_{h}(0)=w_{0 h}, \quad \dot{w}_{h}(0)=w_{1 h} \quad \forall x \in \Omega .
\end{gathered}
$$

In the remaining part of this paper we denote by $\|\cdot\|$ the Euclidean norm in $\mathbb{R}^{m}$ (for various values of the integer $m$ ). The main result of this section is:

Theorem 7.1. The family of systems defined by (7.17)-(7.19) is uniformly exponentially stable, in the sense that there exist constants $M, \alpha, h^{*}>0$ (independent of $h, w_{0 h}$ and $w_{1 h}$ ) such that for all $h \in\left(0, h^{*}\right)$ :

$$
\left\|\dot{w}_{h}(t)\right\|^{2}+\left\|A_{0 h}^{\frac{1}{2}} w_{h}(t)\right\|^{2} \leqslant M \mathrm{e}^{-\alpha t}\left(\left\|w_{1 h}\right\|^{2}+\left\|A_{0 h}^{\frac{1}{2}} w_{0 h}\right\|^{2}\right) \quad \forall t \geqslant 0 .
$$

Proof. Denote $X_{h}=V_{h} \times V_{h}$. Then equations (7.17)-(7.19) can be easily written in the form

$$
\dot{z}_{h}(t)=\widetilde{A}_{h} z_{h}(t), \quad z_{h}(0)=z_{0}
$$

where $\widetilde{A}_{h} \in \mathcal{L}\left(X_{h}\right)$ is defined by (3.17). 
To show the uniform exponential stability, we use Theorem 4.2. By Lemma 3.1, condition $(i)$ in Theorem 4.2 holds true. To prove condition $(i i)$, we use a contradiction argument. Then, for all $n \in \mathbb{N}$, there exist $h_{n} \in\left(0, h^{*}\right), \omega_{n} \in \mathbb{R}, z_{n} \in X_{h_{n}}$ such that

$$
\begin{gathered}
z_{n}=\left[\begin{array}{c}
\varphi_{n} \\
\psi_{n}
\end{array}\right] \in X_{h_{n}} \quad\left\|z_{n}\right\|^{2}=\left\|A_{0 h_{n}}^{\frac{1}{2}} \varphi_{n}\right\|^{2}+\left\|\psi_{n}\right\|^{2}=1 \quad \forall n \in \mathbb{N} \\
i \omega_{n} z_{n}-\widetilde{A}_{h_{n}} z_{n} \rightarrow 0 .
\end{gathered}
$$

By Lemma 3.2, we have

$$
\lim _{n \rightarrow \infty}\left\|\psi_{n}\right\|^{2}=\frac{1}{2}
$$

It can be easily checked (see, for instance, [19]) that the eigenvalues of $A_{0 h}^{\frac{1}{2}}$ are

$$
\widetilde{\lambda}_{p, q, h}=\frac{4}{h^{2}}\left[\sin ^{2}\left(\frac{p h}{2}\right)+\sin ^{2}\left(\frac{q h}{2}\right)\right], \text { for } 1 \leqslant p, q \leqslant \tilde{N} .
$$

The corresponding normalized eigenvectors in $V_{h}$ are

$$
\widetilde{\varphi}_{p, q, h}=\left(\widetilde{\varphi}_{p, q, h}^{j, k}\right)_{1 \leqslant j, k \leqslant \widetilde{N}}, \quad \widetilde{\varphi}_{p, q, h}^{j, k}=\frac{2 h}{\pi} \sin (j p h) \sin (k q h) .
$$

We arrange the sequence $\left(\widetilde{\lambda}_{p, q}\right)_{p, q \in \mathbb{N}^{*}}$ in increasing order to obtain a new sequence $\left(\lambda_{m}\right)_{m \in \mathbb{N}^{*}}$. For $\lambda_{m}=\widetilde{\lambda}_{p, q}$, we set

We set then

$$
\lambda_{m, h}=\widetilde{\lambda}_{p, q, h} \quad \varphi_{m, h}=\widetilde{\varphi}_{p, q, h}, \quad \text { for } 1 \leqslant m \leqslant \widetilde{N}^{2}, 1 \leqslant p, q \leqslant \widetilde{N} .
$$

and for $0<\varepsilon<1$, we define the integer

$$
N(h)=\widetilde{N}^{2}=\left(\frac{\pi}{h}-1\right)^{2}
$$

$$
M\left(h_{n}\right)=\max \left\{m \in\left\{1, \ldots, N\left(h_{n}\right)\right\} \mid h_{n}^{2}\left(\lambda_{m}\right)^{2} \leqslant \varepsilon\right\}, \quad \forall n \in \mathbb{N} .
$$

We can now use arguments similar to those in the proof of Theorem 1.1 by considering the set

$$
\mathcal{F}=\left\{n \in \mathbb{N}|\exists m(n) \in \mathbb{Z}, 1 \leqslant| m(n) \mid \leqslant M\left(h_{n}\right), \text { such that }\left|\omega_{n}-\lambda_{m(n), h_{n}}\right|<\frac{1}{8}\right\} .
$$

Let $\left(c_{m}^{n}\right)_{0<|m| \leqslant N(h)}$ be the coordinates of $z_{n}$ in the basis $\left(\Phi_{m, h}\right)_{0<|m| \leqslant N(h)}$, i.e.,

$$
z_{n}=\sum_{0<|m| \leqslant N\left(h_{n}\right)} c_{m}^{n} \Phi_{m, h}
$$

where $\left(\Phi_{m, h}\right)_{0<|m| \leqslant N(h)}$ are defined by $(3.27)$.

We distinguish two cases:

First case. The set $\mathcal{F}$ is infinite. Then, for the sake of simplicity, we can suppose, without loss of generality, that $\mathcal{F}=\mathbb{N}$. For all $n \in \mathbb{N}$, we introduce then the set $\mathcal{F}_{n}$ (which is never empty, since it always contains $m(n)$ ) defined by

$$
\mathcal{F}_{n}=\left\{m \in \mathbb{Z}|1 \leqslant| m \mid \leqslant M\left(h_{n}\right) \text { and }\left|\omega_{n}-\lambda_{m, h_{n}}\right|<\frac{1}{8}\right\} .
$$


We set then

$$
\widetilde{\psi_{n}}=\frac{1}{\sqrt{2}} \sum_{m \in \mathcal{F}_{n}} c_{m}^{n} \varphi_{m, h_{n}}
$$

One can easily check that $\left(\lambda_{n, h}\right)$ satisfy assumptions of Lemma 3.3 with $\eta=\frac{4}{\pi^{2}}$ and for any $C, \varepsilon>0$. Thus, by the definition of $\mathcal{F}_{n}$, relation (3.34) in Lemma 3.3 implies that

$$
\sum_{m \in\left\{1, \ldots, N\left(h_{n}\right)\right\} \backslash \mathcal{F}_{n}}\left|c_{m}^{n}\right|^{2} \rightarrow 0
$$

Using once again (3.32) and (3.33) in Lemma 3.3, we see that (7.26) implies that

$$
\left\|\psi_{n}-\widetilde{\psi}_{n}\right\| \rightarrow 0
$$

The above relation implies (since $\left(B_{0 h_{n}}^{*}\right)$ is uniformly bounded) that

$$
\left\|B_{0 h_{n}}^{*}\left(\psi_{n}-\widetilde{\psi}_{n}\right)\right\| \rightarrow 0
$$

This relation together with relation (3.21) in Lemma 3.2 show that

$$
\left\|B_{0 h_{n}}^{*} \widetilde{\psi}_{n}\right\| \rightarrow 0
$$

But on the other hand, we have the following result: there exists $\delta>0$ such that for all $n \in \mathbb{N}$, we have

$$
\left\|B_{0 h_{n}}^{*} \widetilde{\psi}_{n}\right\|_{U}^{2} \geqslant \delta \sum_{m \in \mathcal{F}_{n}}\left|c_{m}^{n}\right|^{2} .
$$

The proof of the above relation is given in Lemma 7.2 below.

Gathering (7.26), (7.28) and (7.29), we obtain that $\widetilde{\psi_{n}} \rightarrow 0$ in $H$. By using (7.27), we obtain that $\psi_{n} \rightarrow 0$ which contradicts (7.22).

Second Case. The set $\mathcal{F}$ is finite. Then, for the sake of simplicity, we can suppose, without loss of generality, that $\mathcal{F}$ is empty, i.e., that, for all $n \in \mathbb{N}$, we have:

$$
\left|\omega_{n}-\lambda_{m, h_{n}}\right| \geqslant \frac{1}{8} \text { if } 0<|m| \leqslant M\left(h_{n}\right) .
$$

By using relation (3.34) in Lemma 3.3 and the above relation, we obtain that

$$
\sum_{0<|m| \leqslant M\left(h_{n}\right)}\left|c_{m}^{n}\right|^{2} \rightarrow 0
$$

The above relation, and relations (3.32) and (3.33) in Lemma 3.3 imply that

$$
\psi_{n} \rightarrow 0 \text { in } H,
$$

which contradicts (7.22).

Lemma 7.2. Assume that for all $n \in \mathbb{N}$, there exists an integer $m(n) \in \mathbb{Z}$ such that $\left|\omega_{n}-\lambda_{m(n), h_{n}}\right|<\frac{1}{8}$ (first case in the proof of Th. 7.1). Let $\widetilde{\psi}_{n}$ be defined by (7.25). Then, there exists $\delta>0$ such that for all $n \in \mathbb{N}$, relation (7.29) holds. 
Proof. Without loss of generality, we can assume that the set

$$
\mathcal{F}_{n}=\left\{m \in \mathbb{Z}|1 \leqslant| m \mid \leqslant M\left(h_{n}\right) \text { and }\left|\omega_{n}-\lambda_{m, h_{n}}\right|<\frac{1}{8}\right\}
$$

is included in $\mathbb{N}^{*}$. It will be more convenient to use the following expression of $\widetilde{\psi}_{n}$ :

$$
\widetilde{\psi}_{n}=\sum_{(p, q) \in \mathcal{G}_{n}} c_{p, q}^{n} \widetilde{\varphi}_{p, q, h_{n}}
$$

where $\widetilde{\varphi}_{p, q, h_{n}}$ are defined by $(7.24)$ and

$$
\mathcal{G}_{n}=\left\{(p, q) \in \mathbb{N}^{*} \times \mathbb{N}^{*} \mid h^{2} \widetilde{\lambda}_{p, q}^{2}<\varepsilon \text { and }\left|\omega_{n}-\widetilde{\lambda}_{p, q, h_{n}}\right|<\frac{1}{8}\right\}
$$

is the set described by $(p, q)$ when $m$ describes $\mathcal{F}_{n}$ (recall that $\left.\lambda_{m}=\widetilde{\lambda}_{p, q}=p^{2}+q^{2}\right)$.

$$
\begin{aligned}
\left\|B_{0 h_{n}}^{*} \widetilde{\psi}_{n}\right\|^{2} & =\sum_{j=1}^{\tilde{N}\left(h_{n}\right)} \sum_{k=a\left(h_{n}\right)}^{b\left(h_{n}\right)}\left|\sum_{(p, q) \in \mathcal{G}_{n}} c_{p, q}^{n} \widetilde{\varphi}_{p, q, h_{n}}^{j, k}\right|^{2} \\
& =\sum_{j=1}^{\tilde{N}\left(h_{n}\right)} \sum_{k=a\left(h_{n}\right)}^{b\left(h_{n}\right)} \sum_{(p, q) \in \mathcal{G}_{n}} \sum_{\left(p^{\prime}, q^{\prime}\right) \in \mathcal{G}_{n}} c_{p, q}^{n} \overline{c_{p^{\prime}, q^{\prime}}^{n}} \widetilde{\varphi}_{p, q, h_{n}}^{j, k} \widetilde{\varphi}_{p^{\prime}, q^{\prime}, h_{n}}^{j, k} .
\end{aligned}
$$

Then, one can check that

$$
\sum_{j=1}^{\tilde{N}\left(h_{n}\right)} \widetilde{\varphi}_{p, q, h_{n}}^{j, k} \widetilde{\varphi}_{p^{\prime}, q^{\prime}, h_{n}}^{j, k}=\left\{\begin{array}{cc}
0 & \text { for } p \neq p^{\prime} \\
\frac{2 h_{n}}{\pi} \sin \left(q k h_{n}\right) \sin \left(q^{\prime} k h_{n}\right) & \text { for } p=p^{\prime} .
\end{array}\right.
$$

Furthermore, if $(p, q),\left(p^{\prime}, q^{\prime}\right) \in \mathcal{G}_{n}$ are such that $p=p^{\prime}$, then we necessarily have $q=q^{\prime}$. Indeed, if $(p, q),\left(p^{\prime}, q^{\prime}\right) \in \mathcal{G}_{n}$, then

On the other hand, using the inequality

$$
\left|\widetilde{\lambda}_{p, q, h_{n}}-\widetilde{\lambda}_{p^{\prime}, q^{\prime}, h_{n}}\right|<\frac{1}{4}
$$

$$
x^{2}-\frac{x^{4}}{3} \leqslant \sin ^{2}(x) \leqslant x^{2}, \quad \forall x \in \mathbb{R}
$$

one easily obtains that

$$
\tilde{\lambda}_{p, q}-\frac{\varepsilon}{12} \leqslant \tilde{\lambda}_{p, q, h_{n}} \leqslant \tilde{\lambda}_{p, q}, \quad \forall(p, q) \in \mathcal{G}_{n} .
$$

Gathering (7.31) and the above estimate, we get (since $\varepsilon<1$ ) that

$$
\left|\widetilde{\lambda}_{p, q}-\widetilde{\lambda}_{p^{\prime}, q^{\prime}}\right| \leqslant \frac{1}{2}
$$

Since $\left(\widetilde{\lambda}_{p, q}\right)$ takes only integer values, the above relation implies that $\widetilde{\lambda}_{p, q}=\widetilde{\lambda}_{p^{\prime}, q^{\prime}}$. By using (7.14) we obtain that if $(p, q),\left(p^{\prime}, q^{\prime}\right) \in \mathcal{G}_{n}$ are such that $p=p^{\prime}$ then $q=q^{\prime}$. 
This result, together with (7.30) shows that

$$
\left\|B_{0 h_{n}}^{*} \widetilde{\psi}_{n}\right\|^{2}=\frac{2}{\pi} \sum_{(p, q) \in \mathcal{G}_{n}}\left|c_{p, q}^{n}\right|^{2}\left(\sum_{k=a\left(h_{n}\right)}^{b\left(h_{n}\right)} h_{n} \sin ^{2}\left(q k h_{n}\right)\right) .
$$

We first notice that the term $\mathcal{S}_{n}(q)=\sum_{k=a\left(h_{n}\right)}^{b\left(h_{n}\right)} h_{n} \sin ^{2}\left(q k h_{n}\right)$ can be seen as the Riemann sum corresponding to the integral $\int_{a}^{b} \sin ^{2}(q x) \mathrm{d} x$. It can be easily checked (see, for instance, Lem. A.4. in [3]) that there exists a constant $\delta>0$ such that

On the other hand, we have

$$
\int_{a}^{b} \sin ^{2}(q x) \mathrm{d} x>\delta \quad \forall q \in \mathbb{N}^{*}
$$

$$
\left(\int_{a}^{b} \sin ^{2}(q x) \mathrm{d} x\right)-\mathcal{S}_{n}(q)=\sum_{k=a\left(h_{n}\right)}^{b\left(h_{n}\right)-1} \int_{k h_{n}}^{(k+1) h_{n}}\left(\sin ^{2}(q x)-\sin ^{2}\left(q k h_{n}\right)\right) \mathrm{d} x+h_{n} \sin ^{2}(b q) .
$$

Using the mean value theorem, we obtain that

$$
\left|\left(\int_{a}^{b} \sin ^{2}(q x) \mathrm{d} x\right)-\mathcal{S}_{n}(q)\right| \leqslant(b-a) q h_{n}+h_{n} .
$$

Since $(p, q) \in \mathcal{G}_{n}$, we have $h_{n}^{2} q^{4} \leqslant \varepsilon \leqslant 1$ and thus $q h_{n} \leqslant \sqrt{h_{n}}$ provided $h_{n} \leqslant 1$. Consequently, there exists a constant $C>0$ independent of $q$ and $n$ such that

$$
\left|\left(\int_{a}^{b} \sin ^{2}(q x) \mathrm{d} x\right)-\mathcal{S}_{n}(q)\right| \leqslant C \sqrt{h_{n}}
$$

Using the above relation and (7.33), we immediately get the claimed result (7.29).

\section{REFERENCES}

[1] H.T. Banks and K. Kunisch, The linear regulator problem for parabolic systems. SIAM J. Control Optim. 22 (1984) $684-698$.

[2] H.T. Banks, K. Ito and C. Wang, Exponentially stable approximations of weakly damped wave equations, in Estimation and control of distributed parameter systems (Vorau, 1990), Birkhäuser, Basel, Internat. Ser. Numer. Math. 100 (1991) 1-33.

[3] G. Chen, S.A. Fulling, F.J. Narcowich and S. Sun, Exponential decay of energy of evolution equations with locally distributed damping. SIAM J. Appl. Math. 51 (1991) 266-301.

[4] R.F. Curtain and H. Zwart, An introduction to infinite-dimensional linear systems theory, Texts in Applied Mathematics 21. Springer-Verlag, New York (1995).

[5] E. Fernandez-Cara and E. Zuazua, On the null controllability of the one-dimensional heat equation with BV coefficients. Comput. Appl. Math. 21 (2002) 167-190.

[6] J.S. Gibson, An analysis of optimal modal regulation: convergence and stability. SIAM J. Control Optim. 19 (1981) 686-707.

[7] J.S. Gibson and A. Adamian, Approximation theory for linear-quadratic-Gaussian optimal control of flexible structures. SIAM J. Control Optim. 29 (1991) 1-37.

[8] R. Glowinski, C.H. Li and J.-L. Lions, A numerical approach to the exact boundary controllability of the wave equation. I. Dirichlet controls: description of the numerical methods. Japan J. Appl. Math. 7 (1990) 1-76.

[9] F.L. Huang, Characteristic conditions for exponential stability of linear dynamical systems in Hilbert spaces. Ann. Differ. Equ. 1 (1985) 43-56.

[10] J.A. Infante and E. Zuazua, Boundary observability for the space semi-discretizations of the 1-D wave equation. ESAIM: M2AN 33 (1999) 407-438.

[11] F. Kappel and D. Salamon, An approximation theorem for the algebraic Riccati equation. SIAM J. Control Optim. 28 (1990) $1136-1147$. 
[12] Z. Liu and S. Zheng, Semigroups associated with dissipative systems, Notes in Mathematics 398. Chapman \& Hall/CRC Research, Chapman (1999).

[13] M. Naimark, Linear differential operators. Ungar, New York (1967).

[14] A. Pazy, Semigroups of linear operators and applications to partial differential equations, Springer-Verlag, New York, Appl. Math. Sci. 44 (1983).

[15] J. Prüss, On the spectrum of $C_{0}$-semigroups. Trans. Amer. Math. Soc. 284 (1984) 847-857.

[16] P.-A. Raviart and J.-M. Thomas, Introduction à l'analyse numérique des équations aux dérivées partielles. Dunod, Paris (1998).

[17] G. Strang and G.J. Fix, An analysis of the finite element method. Prentice-Hall Inc., Englewood Cliffs, N.J. Prentice-Hall Series in Automatic Computation (1973).

[18] L.R. Tcheugoué Tébou and E. Zuazua, Uniform exponential long time decay for the space semi-discretization of a locally damped wave equation via an artificial numerical viscosity. Numer. Math. 95 (2003) 563-598.

[19] E. Zuazua, Boundary observability for the finite-difference space semi-discretizations of the 2-D wave equation in the square. J. Math. Pures Appl. 78 (1999) 523-563.

[20] E. Zuazua, Propagation, observation, and control of waves approximated by finite difference methods. SIAM Rev. 47 (2005) $197-243$. 